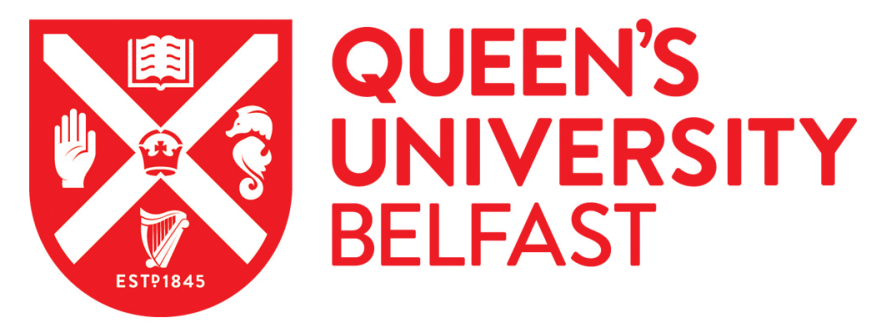

\title{
The cellular and molecular origins of extracellular vesicles released by the helminth pathogen, Fasciola hepatica
}

Bennett, A. P. S., de la Torre Escudero, E., Oliver, N., Huson, K. M., \& Robinson, M. W. (2020). The cellular and molecular origins of extracellular vesicles released by the helminth pathogen, Fasciola hepatica. International Journal for Parasitology. https://doi.org/10.1016/j.ijpara.2020.03.015

Published in:

International Journal for Parasitology

Document Version:

Peer reviewed version

Queen's University Belfast - Research Portal:

Link to publication record in Queen's University Belfast Research Portal

Publisher rights

() 2020 Australian Society for Parasitology Inc.

This manuscript is distributed under a Creative Commons Attribution-NonCommercial-NoDerivs License

(https://creativecommons.org/licenses/by-nc-nd/4.0/), which permits distribution and reproduction for non-commercial purposes, provided the author and source are cited.

\section{General rights}

Copyright for the publications made accessible via the Queen's University Belfast Research Portal is retained by the author(s) and / or other copyright owners and it is a condition of accessing these publications that users recognise and abide by the legal requirements associated with these rights.

Take down policy

The Research Portal is Queen's institutional repository that provides access to Queen's research output. Every effort has been made to ensure that content in the Research Portal does not infringe any person's rights, or applicable UK laws. If you discover content in the Research Portal that you believe breaches copyright or violates any law, please contact openaccess@qub.ac.uk. 
Adam P.S. Bennett, Eduardo de la Torre-Escudero, Nicola A.M. Oliver, Kathryn M. Huson and Mark W. Robinson*

6

School of Biological Sciences, Queen's University Belfast, 19 Chlorine Gardens, Belfast, Northern 8 Ireland.

9

10

* Corresponding author:

11 Mark W. Robinson;

12 School of Biological Sciences,

13 Queen's University Belfast,

1419 Chlorine Gardens

15 Belfast, Northern Ireland

16 Tel: +44 (0)2890972120

17 Email: mark.robinson@qub.ac.uk

18

19

20

Note: Supplementary data associated with this article 
Parasitic helminths secrete extracellular vesicles (EVs) which have potent immunomodulatory effects. Whilst the cargo of EVs has been characterised for many species, we know little about the mechanisms that govern their biogenesis and release. Using antibodies raised against a panel of Fasciola hepatica EV (FhEV) marker proteins, we have identified multiple sites of EV production in the parasite. Discrete immunofluorescence patterns were observed within the gastrodermal cells and tegumental syncytium for different marker proteins whilst the protonephridial (excretory) system and parenchymal-type 2 cells were identified as additional sites of production (or transit) of FhEVs. Ligation was used to mechanically block the oral sucker, excretory pore, or both, to determine the effect on FhEV release from live adult flukes in vitro. This revealed that FhEVs are predominately derived from the gut, whilst the tegument releases EVs to a lesser extent. The data also suggest that the protonephridial system contributes to the small (120K) EV sub-population. Sphingomyelinase (SMase) activity is a key driver of EV biogenesis in mammalian cells and we have previously identified SMases in FhEVs by mass spectrometry. SMase activity associated with isolated FhEVs was susceptible to the chemical inhibitor GW4869 and treatment of adult flukes with GW4869 led to a significant reduction in $120 \mathrm{~K}$ EV release in vitro suggesting that a ceramidedependent mechanism could drive $120 \mathrm{~K} \mathrm{EV}$ formation. In contrast, the release of the larger $15 \mathrm{~K}$ EVs was only moderately impacted, indicating that they form independently of SMase activity. Ultrastructural observation of GW4869-treated $F$. hepatica tissue showed severe disruption to the parenchyma and vacuolation of the tegument, gastrodermal cells and epithelial lining of the excretory ducts. This work establishes that targeted disruption of EV biogenesis and release in helminths is possible and provides proof-of-concept for future studies investigating EV secretion as a target for parasite control. 


\section{Introduction}

The liver fluke, Fasciola hepatica, is a pathogen of medical and veterinary significance and can maintain chronic infections within its mammalian hosts for many years. Like other helminths, it does this by releasing excretory-secretory $(\mathrm{E} / \mathrm{S})$ products to modulate the host immune response. Using mass spectrometry-based proteomics techniques, several groups have built up a comprehensive profile of the E/S material (Cwiklinski et al., 2015; Di Maggio et al., 2016; Morphew et al., 2007; Robinson et al., 2009; Wilson et al., 2011) and identified individual immunomodulatory components (Dalton et al., 2013). However, the discovery of extracellular vesicles (EVs) within the $F$. hepatica secretome has shown that the composition of the E/S products is more complex than originally thought (Marcilla et al., 2012). At least two distinct EV populations, which differ in size and resident protein markers, have been characterised from $F$. hepatica secretions and proteomics analysis has identified a range of known immunomodulators amongst the $F$. hepatica EV (FhEV) cargo proteins (Cwiklinski et al., 2015; Marcilla et al., 2012; de la Torre-Escudero et al., 2019). Indeed, recent work has shown that liver fluke EVs protected against inflammation (independently of $\mathrm{T}$ and $\mathrm{B}$ cells) in a mouse model of colitis (Roig et al., 2018), thus confirming their immunomodulatory capacity.

Given the important immunomodulatory roles of Fasciola EVs (and those of other helminths; reviewed by Zakeri et al., 2018), impairing their release could tip the balance of the hostparasite interaction in favour of the host and enable immune-mediated elimination of the parasite. Although technically challenging, blocking EV release has shown promise in cancer therapy (El Andaloussi et al., 2013), and it was recently demonstrated that blocking Brugia malayi EV release is an action of the anthelmintic ivermectin (Harischandra et al., 2018). As the pharmacologicallyrelevant concentrations of ivermectin used had no direct toxic effect on B. malayi in vitro, it was suggested that preventing EV secretion contributes to the immune-mediated killing of the parasite (Harischandra et al., 2018). Based on this precedent, developing therapeutics to inhibit the release 
of FhEVs could have anthelmintic potential. However, this approach requires a better understanding of the mechanism(s) driving FhEV biogenesis and release.

Some insight into EV biogenesis in helminths has been provided by proteomics studies. Orthologues of sphingomyelinases (SMases) and endosomal sorting complex required for transport (ESCRT) proteins, the major players in multivesicular body-driven exosome formation in mammalian cells (Kowal et al., 2014; Trajkovic et al., 2008), were identified in FhEVs, suggesting that similar mechanisms may operate in the parasite (Cwiklinski et al., 2015; de la Torre-Escudero et al., 2019, 2016). However, the molecular pathways driving FhEV formation, their mechanisms of release, and their sites of secretion from the parasite remain largely unknown.

In this study, we have used a panel of antibodies, raised against proteins enriched in FhEVs, to determine sites of EV production in adult fluke tissue sections. Our data reveal discrete localisation patterns in the tegument, gut and protonephridial system, and further immunostaining of vesicular structures within parenchymal cells suggests that EVs may also be produced in this tissue before being trafficked to other sites for extra-corporeal release. Ligation of adult flukes in vitro showed that, whilst EVs are predominately derived from the gastrointestinal tract, some are released from the protonephridial system, via the excretory pore, whilst others may be shed directly from the tegument. We also demonstrate that SMase activity associated with FhEVs is susceptible to the chemical inhibitor GW4869. Treatment of flukes with GW4869 suppressed secretion of 120K EVs in vitro and led to major disruption of parenchymal cell ultrastructure. Collectively, our data show that targeted disruption of helminth EV biogenesis and release is possible which provides a framework for future studies using in vivo infection models.

\section{Materials and Methods}

\section{Immunolocalisation}

Adult $F$. hepatica were collected from the bile ducts of sheep immediately after slaughter at a local abattoir (Dungannon, Northern Ireland). Worms were fixed for $4 \mathrm{~h}$ in $4 \%$ paraformaldehyde in 0.1 
M PBS (Sigma-Aldrich) and subsequently embedded in JB-4 resin (Sigma-Aldrich) according to the manufacturer's instructions. Semi-thin $(2 \mu \mathrm{m})$ sections were cut on a pyramitome and mounted on clean glass slides. For immunofluorescence, tissue sections were washed in PBS then incubated overnight in primary antibody in antibody diluent (AbD: PBS containing 0.2\% (v/v) Triton X-100) at room temperature $\left(18-21^{\circ} \mathrm{C}\right)$. For details of the primary antibodies, including their target peptides, working concentrations and source, see Supplementary Table 1. As a negative control, tissue sections were also incubated with pre-immune serum in $\mathrm{AbD}$. The sections were then washed three times in $\mathrm{AbD}$ before incubation in an appropriate secondary antibody-fluorophore conjugate (goat anti-rabbit IgG-FITC or goat anti-mouse IgG-TRITC, Sigma-Aldrich) at a 1:200 dilution in $\mathrm{AbD}$ for $1 \mathrm{~h}$ at room temperature. Following four washes in PBS, the sections were mounted in glycerol containing 10\% (v/v) PBS and 0.1 M propyl gallate (Sigma-Aldrich) then viewed under a Leica DM2500 fluorescent microscope.

\section{Ligation and in vitro culture of adult F. hepatica}

Adult flukes were obtained from the bile ducts of sheep immediately after slaughter. After thoroughly washing the flukes in PBS containing $0.1 \%(\mathrm{w} / \mathrm{v})$ glucose at $37^{\circ} \mathrm{C}$, those that had regurgitated their gut contents were selected for ligation using the method of Rohrbacher (1957). Briefly, flukes were ligated around the oral sucker or the excretory pore, or both using sterile surgical thread (5-0 Prolene ${ }^{\circledR}$ Polypropylene Suture, Ethicon). Any flukes that were damaged by the ligation process were discarded. Flukes were ligated in groups of 30, in biological triplicate (with non-ligated flukes acting as control) and maintained at 1 worm/ml in RPMI-1640 culture medium containing $0.1 \%(\mathrm{w} / \mathrm{v})$ glucose, $100 \mathrm{U} / \mathrm{ml}$ penicillin and $100 \mu \mathrm{g} / \mathrm{ml}$ streptomycin (SigmaAldrich) for $3 \mathrm{~h}$ at $37^{\circ} \mathrm{C}$ to collect EVs. During culture, the integrity and survival of the flukes was monitored visually. The wet weight of each group was used to normalise downstream protein concentration. After $3 \mathrm{~h}$ incubation, EVs were isolated from the culture media by differential centrifugation as described by de la Torre-Escudero et al. (2019) to determine the effect of ligation 
on the release of the $15 \mathrm{~K} \mathrm{EVs}$ (post-15,000 x $g$ pellet) and $120 \mathrm{~K}$ EVs (post-120,000 x $g$ pellet) characterised by Cwiklinski et al. (2015). Both EV pellets were resuspended in filter-sterilised PBS, snap-frozen and stored at $-80^{\circ} \mathrm{C}$ until further analysis.

\section{Determining particle and protein concentrations}

The protein concentration of lysed EVs was determined using the Pierce ${ }^{\mathrm{TM}}$ BCA Protein Assay Kit (ThermoFisher Scientific). Nanoparticle tracking analysis (NTA) was carried out using the NanoSight NS300 system (Malvern Instruments Ltd, United Kingdom) equipped with a $638 \mathrm{~nm}$ laser and NanoSight NTA software version 3.2. EVs that had undergone a single freeze-thaw cycle were diluted in filter-sterilised PBS and five 1 min videos of each sample were recorded with optimised set parameters and a $10 \mathrm{~s}$ delay between recordings. Data for each sample are presented as the mean of the five recordings. All measurements for each experimental group were performed in biological triplicate and the dilution factor for each sample was set to account for the wet weight of flukes in each replicate.

\section{Immunoblot analysis of $F$. hepatica extracellular vesicles}

Antibodies raised against FhEV proteins (de la Torre-Escudero et al., 2019) were used as markers to determine the effect of ligation on the release of EVs from adult worms during in vitro culture. For immunoblotting, EVs normalised by wet weight of the cultured flukes $(2 \mu 115 \mathrm{~K} \mathrm{EVs} / \mathrm{g}$ fluke or 4 $\mu \mathrm{l} 120 \mathrm{~K} \mathrm{EVs} / \mathrm{g}$ fluke) were run on reducing NuPAGE Novex 4-12\% Bis-Tris gels (ThermoFisher Scientific) and transferred to nitrocellulose membranes (GE Healthcare) at $30 \mathrm{~V}$ for 40 minutes. Following transfer, the membrane was incubated in blocking solution (TBST: $20 \mathrm{mM}$ Tris-HCl, 150 $\mathrm{mM} \mathrm{NaCl}$, and $1 \%$ Tween-20, $\mathrm{pH} 7.6$ ) containing 5\% skimmed milk for $1 \mathrm{~h}$ at room temperature. Blots were then probed with primary antibodies (for details see Supplementary Table 1) diluted in TBST containing $1 \%$ skimmed milk for $1 \mathrm{~h}$ at room temperature. After washing in TBST (3 x 10 minutes), blots were incubated with an appropriate alkaline phosphatase-conjugated IgG secondary 
antibody (Sigma-Aldrich) for $1 \mathrm{~h}$ at room temperature before detection using the BCIP/NBT substrate (Sigma-Aldrich). Blots were imaged using the Syngene G:BOX F3 system (Synoptics Ltd, UK) and densitometric quantification was performed with ImageJ software version 1.48.

\section{Statistical analysis}

Data are presented as the mean of three biological replicates, \pm standard error. Significance values were calculated using a two-tailed t-test with two-sample unequal variance. Statistical analysis was performed using Excel version 16.0.9126.2295 (Microsoft). A p value $<0.05$ was considered significant.

\section{Bioinformatics analysis of $F$. hepatica sphingomyelinases}

The evolutionary relationship of Homo sapiens (Hs) SMases (HsSMPD1, P17405; HsSMPD2, O60906; HsSMPD3, Q9NY59; HsSMPDL3a, Q92484; HsSMPDL3b, Q92485) and F. hepatica orthologues (FhSMPD2, BN1106_s7135B000046; FhSMPDL3 ${ }^{159}$, BN1106_S1285B000159; FhSMPDL3 ${ }^{138}$, BN1106_S3568B000138) was inferred using the neighbour-joining method (Saitou and Nei, 1987). The superscript number in FhSMPDL3 ${ }^{159 / 138}$ refers to the last three digits of the protein's identifier to distinguish it from the other isoform. The bootstrap consensus tree inferred from 1000 replicates was taken to represent the evolutionary history of the taxa analysed (Felsenstein, 1985). Branches corresponding to partitions reproduced in less than $50 \%$ bootstrap replicates were collapsed. The evolutionary distances were computed using the Poisson correction method (Zuckerandl and Pauling, 1965) and are in the units of the number of amino acid substitutions per site. All positions containing gaps and missing data were eliminated. There were a total of 284 positions in the final dataset. Evolutionary analyses were conducted in MEGA6 (Tamura et al., 2013). The domain organisation of $F$. hepatica (Fh)SMases was determined using InterPro (https://www.ebi.ac.uk/interpro/). 
GW4869 inhibition assay

The susceptibility of native SMase activity associated with adult FhEVs to GW4869 was assayed using the Amplex Red Sphingomyelinase Assay Kit (ThermoFisher Scientific) according to the manufacturer's instructions. Four $\mu \mathrm{g}$ of $15 \mathrm{~K}$ EVs or $2 \mu \mathrm{g} 120 \mathrm{~K}$ EVs were incubated with $0-50 \mu \mathrm{M}$ GW4869 (dissolved in DMSO). DMSO alone was added to some EVs as a vehicle control. Assays, performed in biological triplicate, were incubated for $15 \mathrm{~min}(120 \mathrm{~K} \mathrm{EVs})$ or $1 \mathrm{~h}(15 \mathrm{~K} \mathrm{EVs})$ in the dark at $37^{\circ} \mathrm{C}$ before fluorescence measurements were made using a POLARstar Omega Spectrophotometer (BMG LABTECH, Germany) (excitation 544 nm/emission $590 \mathrm{~nm}$ ).

\section{In vitro treatment of $F$. hepatica with GW4869}

Adult flukes were obtained from sheep, as described above, and washed thoroughly in PBS containing $0.1 \%(\mathrm{w} / \mathrm{v})$ glucose at $37^{\circ} \mathrm{C}$. Those that had regurgitated their gut contents were incubated in triplicate groups of 30 at 2 worms $/ \mathrm{ml}$ in RPMI-1640 culture medium containing $0.1 \%$ (w/v) glucose, $100 \mathrm{U} / \mathrm{ml}$ penicillin, $100 \mu \mathrm{g} / \mathrm{ml}$ streptomycin (Sigma-Aldrich) and either $25 \mu \mathrm{M}$ GW4869 (Sigma-Aldrich) or $0.5 \%$ DMSO (vehicle control) for $3 \mathrm{~h}$ at $37^{\circ} \mathrm{C}$. The flukes were then placed in fresh media and incubated for a further $3 \mathrm{~h}$ to collect parasite secretions. The wet weight of each group was determined and used to normalise downstream protein concentrations. During culture the integrity and survival of the flukes was monitored visually. Following culture the EVs were collected and quantified by protein concentration, NTA and immunoblotting as described above.

\section{Processing of adult F. hepatica for microscopy}

Following in vitro culture, control and GW4869-treated flukes were fixed and processed for transmission electron microscopy as previously described (Robinson et al., 2002). Briefly, whole worms were fixed in $4 \%(\mathrm{w} / \mathrm{v})$ glutaraldehyde in $0.1 \mathrm{M}$ sodium cacodylate buffer ( $\mathrm{pH} 7.4)$ containing (w/v) 3\% sucrose for $30 \mathrm{~min}$. Worms were then dissected, and mid-body sections were 
placed in fresh fixative for $4 \mathrm{~h}$. Post-fixation, the tissue was washed in $0.1 \mathrm{M}$ sodium cacodylate buffer ( $\mathrm{pH} 7.4$ ) containing 3\% (w/v) sucrose for $24 \mathrm{~h}$. Fixed tissue was then placed in $1 \%$ osmium tetroxide for $1 \mathrm{~h}$ and washed again in $0.1 \mathrm{M}$ sodium cacodylate buffer $(\mathrm{pH} 7.4)$ containing 3\% (w/v) sucrose for $24 \mathrm{~h}$. The tissue was dehydrated in ethanol before infiltration and embedding in Agar 100 epoxy resin (Agar Scientific, UK). Ultrathin sections $(80 \mathrm{~nm})$ were mounted on uncoated copper grids then stained with uranyl acetate and aqueous lead citrate at the Electron Microscopy facility at the Conway Institute, University College Dublin. The grids were viewed using a Jeol 1400 TEM operating at $80 \mathrm{kV}$ at the Agri-Food and Biosciences Institute (Stormont, Northern Ireland).

\section{Results}

F. hepatica extracellular vesicle markers localise to the gut, tegument, protonephridial ducts and parenchymal tissue

To determine the sites of FhEV production, trafficking and release, a panel of antibodies raised against proteins found to be enriched in FhEVs by mass spectrometry (Cwiklinski et al., 2015; de la Torre-Escudero et al., 2019) were used to probe adult $F$. hepatica tissue sections. These targets included orthologues of known EV biogenesis proteins such as TSG101 and ALIX which are involved in the ESCRT-dependent pathway (Christ et al., 2017); Ral-A which is associated with a non-canonical form of the ESCRT pathway that utilises only a limited selection of ESCRT components to form exosomes (Hyenne et al., 2016); and SMases which operate independently of ESCRT in EV biogenesis (van Niel et al., 2018). Other antibodies targeted the fluke-specific proteins tegumental antigen 1 (FhTeg1), T cell immunomodulatory protein (FhTIP) and DM9 domain-containing protein (FhDM9) (Cwiklinski et al., 2015), whilst $\alpha$-tubulin was used as a marker of EVs that pellet at low centrifugal forces (Menck et al., 2017).

Antibodies against several FhEV markers labelled the gastrodermal cells, albeit with discrete localisation patterns (Fig. 1). F. hepatica gastrodermal cells line the gut caeca and have a 
pyramidal shape (Fig. 1A) and showed no specific immunofluorescence when sections were probed with pre-immune control serum (Fig. 1B). However, immunostaining with the anti-FhALIX, FhDM9 and FhTIP antibodies revealed foci of immunofluorescence concentrated towards the gastrodermal cell apices that become more dispersed towards the basolateral membrane. Points of immunofluorescence were also visible in rear-cell extensions that penetrated backwards into the parenchymal tissue (Fig. 1C, F-G). Similar immunofluorescent puncta were observed clustered in and around parenchymal cells underlying the gastrodermis for FhALIX, FhDM9, FhTIP and FhSMPDL3 $^{159}$ (Fig. 1C, E, F-G). Only FhDM9 showed immunopositivity in the gut lumen where larger globular structures were labelled (Fig. 1F). Faint FhSMPD2 and FhSMPDL3 ${ }^{159}$-positive immunofluorescence was seen in the gastrodermal cell lamellae (Fig. 1D-E), and discrete puncta of immunofluorescence were also observed in this region for tyrosinated $\alpha$-tubulin (Fig. $1 \mathrm{H}$ ). These proteins showed contrasting immunostaining in the cell bodies, however, with FhSMPD2 exhibiting diffuse immunofluorescence, whilst labelling was absent for FhSMPDL $3^{159}$ and tyrosinated $\alpha$ tubulin (Fig. 1D-E, H).

The tegumental syncytium of adult $F$. hepatica is the major barrier between the parasite and the host environment and encloses the parenchymal tissue and internal organs (Threadgold, 1963; Fig. 2A). No specific immunofluorescence was observed in the tegument in sections probed with pre-immune serum (Fig. 2B), but the tegumental syncytium was labelled by antibodies targeting FhSMPDL ${ }^{159}$, FhDM9 and FhTeg1 (Fig. 2C-E). This immunofluorescence was concentrated at the base of the syncytium, but FhDM9 and FhTeg1 were also localised to the apical plasma membrane. Additionally, FhDM9 and FhTeg1 displayed a more granular immunofluorescence pattern than FhSMPDL ${ }^{159}$, with FhTeg1 present in discrete puncta that were similarly visible in the cytoplasmic connections that join the syncytium to the underlying tegumental cell bodies. FhSMPDL $3^{159}$ also showed intense immunostaining in the cytoplasmic connections, along with fainter immunofluorescence around the periphery of tegumental cell bodies (Fig. 2C). The tegumental cell bodies themselves displayed diffuse FhDM9 and FhTeg1 immunoreactivity 
punctured by larger foci of immunofluorescence within the cytoplasm (Fig. 2D-E). This was more intense for FhTeg1 and consistent with pockets of $\mathrm{T} 1$ secretory bodies arising from Golgi complexes (Hanna, 1980). The anti-FhDM9 antibody also labelled vesicular structures situated in large clusters within parenchymal cells or dispersed below the tegumental syncytium (Fig. 2D).

The protonephridial (excretory) system is a major source of lipid-expulsion by the parasite (Burren et al., 1967) and consists of a network of ducts lined by a cellular epithelium (Wilson and Webster, 1974; Fig. 3A). Whilst no specific immunofluorescence was seen in these areas in sections probed with pre-immune control serum (Fig. 3B), both the protonephridial duct epithelium and pockets of vesicular structures in sub-protonephridial parenchymal cells were labelled by antibodies targeting FhTSG101, FhALIX, FhRal-A, FhSMPD2, and FhDM9 (Fig. 3C-G). The immunolocalisation pattern within the protonephridial duct epithelium, however, varied with FhTSG101 and FhSMPD2 showing diffuse immunofluorescence (Fig. 3C and F), FhALIX and FhRal-A displaying a granular immunofluorescence pattern (Fig. 3D and E) (with FhALIX resembling its gastrodermal cell localisation pattern seen in Fig. 1C), whilst FhDM9-positive immunofluorescence was situated mainly in perinuclear regions of the duct epithelial cells (Fig. $3 G)$.

To investigate the labelling of parenchymal cells by antibodies targeting FhEV markers, sections that were first used for immunolocalisation were subsequently stained with toluidine blue to help classify the labelled cells. The immunopositive vesicular structures, seen clustered within parenchymal cells, stained dark blue which indicates that they are basophilic (Fig. 4). The cytoplasm of some of these cells displayed slight basophilia and the vesicular structures could occupy the majority of the cytoplasm (Fig. 4). This cell type was commonly located in subgastrodermal and sub-tegumental regions and resembled the PC2-type parenchymal cells in $F$. gigantica described by Pankao et al. (Pankao et al., 2006).

\section{Ligation of the oral sucker prevents the release of $F$. hepatica extracellular vesicles in vitro}


To determine the principal route(s) of export of FhEVs, adult $F$. hepatica were ligated at the oral sucker (OSL), excretory pore (EPL), or both (double ligated, DL), and then maintained for $3 \mathrm{~h}$ in vitro. The flukes remained motile following incubation and representative images of the ligated flukes are shown in Fig. 5. In comparison to the prominent $15 \mathrm{~K}$ EV pellet recovered from nonligated control fluke culture media, the OSL and DL 15K EV pellets were almost invisible (Fig. 5). In contrast, the $15 \mathrm{~K} \mathrm{EV}$ pellets recovered from the EPL flukes looked similar to the controls (Fig. 5). The $120 \mathrm{~K} \mathrm{EV}$ pellets are typically too faint to be compared visually.

Varied analyses were performed to determine the protein concentration, particle concentration and particle size of $15 \mathrm{~K}$ and $120 \mathrm{~K}$ EVs collected from control and ligated flukes. OSL resulted in a $31 \%$ reduction in $15 \mathrm{~K} \mathrm{EV}$ protein concentration and a $25 \%$ reduction in $15 \mathrm{~K} \mathrm{EV}$ particle concentration compared to control $15 \mathrm{~K}$ EVs, although these reductions were not statistically significant (Supplementary Fig. S1A-B). Additionally, it was estimated that OSL flukes released protein associated with $15 \mathrm{~K}$ EVs at a rate of approximately $790 \mathrm{ng} / \mathrm{fluke} / \mathrm{h}$ and $15 \mathrm{~K} \mathrm{EV}$ particles at $\sim 4.27 \times 10^{8}$ particles/fluke/h, as opposed to the control flukes which released approximately $1150 \mathrm{ng} /$ fluke/h and $\sim 5.69 \times 10^{8}$ particles/fluke/h. The quantity of protein per particle was similar, averaging around 2.0 femtograms (fg)/particle in both the control and OSL $15 \mathrm{~K} E V$ pellets, and there was no significant difference in the average size of $15 \mathrm{~K}$ EVs collected from OSL flukes $(224 \mathrm{~nm})$ compared to the control 15K EVs $(219 \mathrm{~nm})$. Western blotting was used to determine whether ligation affected the release of EVs bearing different protein markers. Consistent with their association with the gut, FhSMPDL $3^{159}$, tyrosinated $\alpha$-tubulin, cathepsin L1 (FhCL1) and CD63 receptor (FhCD63rec) showed 65 - 93\% reduction within the 15K EV population after OSL, with all but FhCL1 being statistically significant (Fig. 6A). Collectively, these experiments demonstrate that OSL had a marked impact on the release of $15 \mathrm{~K}$ EVs from adult flukes in vitro.

OSL had an even greater effect on the release of $120 \mathrm{~K}$ EVs, with $>50 \%$ reduction $(\mathrm{p}<$ 0.01 ) in protein concentration and $>65 \%$ reduction in particle concentration (Supplementary Fig. 
S1A-B) compared to the control 120K EVs. Additionally, it was estimated that OSL flukes released protein associated with $120 \mathrm{~K} \mathrm{EVs}$ at a rate of $70 \mathrm{ng} /$ fluke/h and $120 \mathrm{~K} \mathrm{EV}$ particles at $2.58 \times 10^{7}$ particles/fluke/h, as opposed to the control flukes which released approximately $140 \mathrm{ng} / \mathrm{fluke} / \mathrm{h}$ and $7.90 \times 10^{7}$ particles/fluke/h. Again, the protein content of control and OSL 120K EVs was $~ 2.0$ $\mathrm{fg} /$ particle and there was no significant difference in the particle sizes or size distribution between them. The reductions in protein and particle concentration were similarly reflected in Western blots (Fig. 6B) with the release of FhSMPDL $3^{159}$-positive and FhCD63rec-positive 120K EVs significantly reduced by OSL $(\mathrm{p}<0.01$ and $\mathrm{p}<0.05$, respectively). These findings demonstrate that OSL substantially reduced the release of $120 \mathrm{~K}$ EVs by F. hepatica in vitro.

\section{Excretory pore ligation of $F$. hepatica has differing effects on the release of $E V$ populations in vitro}

EPL showed an increase (though not significant) of $28 \%$ and $25 \%$ in $15 \mathrm{~K}$ EV protein and particle concentrations, respectively, in comparison to control 15K EVs (Supplementary Fig. S1C-D). Accordingly, it was estimated that EPL flukes released protein associated with $15 \mathrm{~K} \mathrm{EVs}$ at a rate of $650 \mathrm{ng} /$ fluke/h and $15 \mathrm{~K} \mathrm{EV}$ particles at $\sim 4.23 \times 10^{9}$ particles/fluke/h, compared to the control flukes which released approximately $590 \mathrm{ng} /$ fluke/h and $\sim 3.73$ x $10^{9}$ particles/fluke/h. There were no differences in the sizes of control and EPL 15K EVs (both averaging approximately $235 \mathrm{~nm}$ ), the protein content $(0.16 \mathrm{fg} /$ particle on average $)$ and they also displayed similar size distributions. In accordance with the protein and particle concentrations, increases in the levels of the FhEV marker proteins tyrosinated $\alpha$-tubulin, FhCL1, and FhCD63rec were observed within the EPL 15K EV preparation relative to the control $15 \mathrm{~K}$ EVs (Fig. 6C), indicating that EPL increases the number of $15 \mathrm{~K}$ EVs derived from the gut.

In contrast to the $15 \mathrm{~K} \mathrm{EVs}$, EPL caused a decrease in $120 \mathrm{~K} \mathrm{EV}$ protein and particle concentrations by approximately 40 - 50\% (Supplementary Fig. S1C-D). The EPL flukes released protein associated with $120 \mathrm{~K} \mathrm{EVs}$ at a rate of approximately $90 \mathrm{ng} / \mathrm{fluke} / \mathrm{h}$ and $120 \mathrm{~K} \mathrm{EV}$ particles at $\sim 1.55 \times 10^{9}$ particles/fluke/h, as opposed to the control flukes which released approximately 170 
ng/fluke/h and $\sim 3.09 \times 10^{9}$ particles/fluke/h. There were no differences in the sizes of control and EPL 120K EVs (both averaged between $163-164 \mathrm{~nm}$ ), the protein content (both $0.05-0.06$ fg/particle), and both EPL and control 120K EVs displayed similar size distributions. Although there was some variation between replicates, densitometric analysis of Western blots showed that the average band intensities for FhTSG101, FhSMPDL3 ${ }^{159}$ and FhCD63rec were reduced in the EPL 120K EV population relative to control 120K EVs (Fig. 6D), and a significant reduction (p < $0.05)$ in $120 \mathrm{~K}$ EVs positive for FhRal-A was observed.

\section{Ligating both the oral sucker and the excretory pore of F. hepatica (double ligation) blocks $120 \mathrm{~K}$} EV release in vitro and significantly alters $15 \mathrm{~K}$ EV composition

Remarkably, DL had no effect on protein concentration, particle concentration or size distribution of $15 \mathrm{~K}$ EVs compared to the controls (Supplementary Fig. S1E-F). It was also estimated that control and DL flukes released $15 \mathrm{~K} \mathrm{EV}$-associated protein and particles at approximately the same rate: $590 \mathrm{ng} / \mathrm{fluke} / \mathrm{h}$ and $3.58-3.73 \times 10^{9}$ particles/fluke/h. Equally, there was no difference in $15 \mathrm{~K}$ EV protein content between the control and DL flukes (both $\sim 0.16 \mathrm{fg} /$ particle). In contrast, Western blotting showed that the EV markers tyrosinated $\alpha$-tubulin, FhCL1, FhCD63rec and FhSMPDL3 ${ }^{159}$ displayed a $40-75 \%$ reduction in band intensity (Fig. 6E), indicating that (similarly to OSL) DL markedly reduced the release of $15 \mathrm{~K}$ EVs carrying these proteins from the gut.

In contrast to the effect on $15 \mathrm{~K}$ EVs, DL reduced $120 \mathrm{~K}$ EV protein concentration by $>70 \%$ (Supplementary Fig. S1E), and the particle concentration was reduced by $\sim 90 \%$ compared to the control 120K EVs (Supplementary Fig. S1F). Additionally, it was estimated that control flukes released protein associated with $120 \mathrm{~K} \mathrm{EVs}$ at a rate of $170 \mathrm{ng} /$ fluke/h and $120 \mathrm{~K} \mathrm{EV}$ particles at 3.09 x $10^{9}$ particles/fluke/h, whilst the rates of $120 \mathrm{~K} \mathrm{EV} \mathrm{release} \mathrm{by} \mathrm{DL} \mathrm{flukes} \mathrm{were} \sim 50 \mathrm{ng} /$ fluke/h and $3.35 \times 10^{8}$ particles/fluke/h. The estimated protein content of DL $120 \mathrm{~K}$ EVs was almost three times greater than control $120 \mathrm{~K}$ EVs $(0.14 \mathrm{fg} /$ particle compared to $0.05 \mathrm{fg} /$ particle), although no significant difference in average size of the EVs was observed (both $161-163 \mathrm{~nm}$ ). Western 
blotting similarly demonstrated a decrease of over 50\% in the levels of FhTSG101, FhSMPDL3 ${ }^{159}$, FhCD63rec, and FhRal-A in the DL 120K EV population (Fig. 6F). These findings indicate that DL significantly inhibited the release of $120 \mathrm{~K}$ EVs by $F$. hepatica in vitro.

\section{Phylogenetic and sequence analysis of F. hepatica sphingomyelinases}

The conversion of sphingomyelin to ceramide by SMases is a key driver of ESCRT-independent EV biogenesis (Verderio et al., 2018) and acid and neutral SMase family members (characterised by their pH optima) have both been implicated this process (Gradilla et al., 2014; Menck et al., 2017; Trajkovic et al., 2008; Truman et al., 2012). BLAST searches indicated that only two acid-like SMases and one neutral SMase are conserved in F. hepatica. Phylogenetic analysis revealed that human SMases and F. hepatica orthologues group by functional similarity, rather than into speciesspecific clades (Supplementary Fig. S2A). Whilst FhSMPD2 grouped with its neutral SMase family orthologue (HsSMPD2), FhSMPDL $3^{159}$ and FhSMPDL $3^{138}$ formed their own discrete cluster within the acid-like SMase family, set apart from HsSMPD1 and HsSMPDL3a/b. Despite their segregation, the predicted domain architectures of FhSMPDL $3{ }^{159}$ and FhSMPDL $3^{138}$ showed that they were more similar to HsSMPDL3a/b (Supplementary Fig. S2B): both have a predicted Nterminal signal peptide and metallo-phosphatase catalytic domain, but lack the N-terminal saposin domain of HsSMPD1 (Gorelik et al., 2016b). FhSMPDL $3^{138}$ also had an additional predicted transmembrane region but neither protein was predicted to have a GPI anchor which is a feature of SMPDL3b (Heinz et al., 2015). FhSMPD2 displayed the same domain architecture as HsSMPD2 (Supplementary Fig. S2B), including two transmembrane regions which are essential for activity (Tomiuk et al., 2000). Catalytic residues were conserved in FhSMPD2, including Asp ${ }^{111}$ and Lys ${ }^{116}$ of the DK switch, a motif involved in both catalysis and interactions with the neutral SMase inhibitor GW4869 (Airola et al., 2017). In contrast, fewer functionally-important residues were conserved in the fluke acid-like SMases, particularly in FhSMPDL3 ${ }^{159}$ which lacked residues such as $\mathrm{His}^{97}$ and Lys ${ }^{140}$ which are important in substrate recognition and binding (Gorelik et al., 2016a). 
FhSMPDL $3^{159}$ and FhSMPDL ${ }^{138}$ also lacked key $N$-linked glycosylation sites required for HsSMPDL3a function and, like in HsSMPDL3a/b, only the terminal Asn residues of the acid SMase (SMPD1) consensus motif NXXXCXXXN (where X is any amino acid) were conserved.

GW4869 inhibits F. hepatica sphingomyelinase activity and suppresses release of extracellular vesicles in vitro

To determine whether native FhSMase activity associated with EVs was sensitive to GW4869, an inhibitor of mammalian neutral SMases (Trajkovic et al., 2008), FhEVs were treated with increasing concentrations of the inhibitor (Fig. 7A and Supplementary Fig. S3A). Whilst $5 \mu \mathrm{M}$ GW4869 had no significant effect on SMase activity, $25 \mu \mathrm{M}$ GW4869 decreased 15K and 120Kassociated SMase activity by $27 \%$ and $35 \%$, respectively. Treatment with $50 \mu \mathrm{M}$ GW4869 decreased 120K-associated SMase activity by 64\% (Fig. 7A), but this increased concentration had no additional inhibitory effect on 15K EV-associated activity (Supplementary Fig. S3A).

As FhSMase activity was sensitive to GW4869, adult $F$. hepatica were maintained for $6 \mathrm{~h}$ in vitro in the presence of $25 \mu \mathrm{M}$ GW4869 (or $0.5 \%$ DMSO as a vehicle control) to determine the effect on FhEV release. At the end of the incubation period, the flukes remained motile, but GW4869 treatment resulted in a significant $(\mathrm{p}<0.05) 25 \%$ reduction in $15 \mathrm{~K}$ EV protein concentration and a $24 \%$ reduction in $15 \mathrm{~K} \mathrm{EV}$ particle concentration compared to control $15 \mathrm{~K}$ EVs (Supplementary Fig. S3B-C). Additionally, GW4869-treated flukes released protein associated with $15 \mathrm{~K} \mathrm{EVs}$ at a rate of $470 \mathrm{ng} / \mathrm{fluke} / \mathrm{h}$ and $15 \mathrm{~K} \mathrm{EV}$ particles at $\sim 2.04 \times 10^{9}$ particles/fluke/h, as opposed to the control flukes which released $630 \mathrm{ng} / \mathrm{fluke} / \mathrm{h}$ and $\sim 2.69 \times 10^{9}$ particles/fluke/h. GW4869 treatment had no effect on the quantity of protein per particle $(0.23 \mathrm{fg} /$ particle for $15 \mathrm{~K}$ EVs derived from both treated and untreated flukes) or on the average size of 15K EVs ( $255 \mathrm{~nm})$ which both had similar size distributions. Western blotting was also used to assess whether GW4869 treatment could affect the release of FhEVs bearing particular protein markers. As shown in Supplementary Fig. S3D, the levels of EV markers tyrosinated $\alpha$-tubulin, FhCD63rec, FhCL1, 
and FhSMPDL $3^{159}$ were reduced by $16-31 \%$, although this was only significant $(\mathrm{p}<0.05)$ for FhSMPDL ${ }^{159}$. Collectively, these results demonstrate that GW4869 treatment had a measurable, but modest, impact on $15 \mathrm{~K} \mathrm{EV}$ release from adult flukes in vitro.

In contrast, GW4869 substantially impacted the release of $120 \mathrm{~K}$ EVs, with a $55 \%$ reduction $(\mathrm{p}<0.01)$ in protein concentration $($ Fig. 7B) and an $80 \%(\mathrm{p}<0.05)$ reduction in particle concentration (Fig. 7C) compared to $120 \mathrm{~K} \mathrm{EVs} \mathrm{recovered} \mathrm{from} \mathrm{vehicle-treated} \mathrm{flukes.} \mathrm{Furthermore,}$ GW4869-treated flukes released protein associated with $120 \mathrm{~K} \mathrm{EVs}$ at a rate of approximately 17 ng/fluke/h and $120 \mathrm{~K} \mathrm{EV} \mathrm{particles} \mathrm{at} ~ 3.24 \times 10^{7}$ particles/fluke/h, compared to the control flukes which released approximately $38 \mathrm{ng} /$ fluke/h and $\sim 1.59 \times 10^{8}$ particles/fluke/h. The protein content of the $120 \mathrm{~K} \mathrm{EVs}$ was $0.24 \mathrm{fg} /$ particle for control $120 \mathrm{~K} \mathrm{EVs} \mathrm{compared} \mathrm{with} 0.53 \mathrm{fg} / \mathrm{particle}$ for the 120K EVs from GW4869-treated flukes which could indicate the presence of more protein aggregates in the $120 \mathrm{~K}$ EV pellet derived from GW4869-treated flukes. There was no significant difference in $120 \mathrm{~K}$ EV particle size or size distribution. Western blotting showed an $86 \%(\mathrm{p}<0.05)$ and $89 \%(\mathrm{p}<0.001)$ reduction in the release of FhSMPDL3 ${ }^{159}$-positive and FhCD63rec-positive 120K EVs (Fig. 7D), respectively, reflecting the change in particle concentration rather than total protein concentration. These findings show that GW4869 treatment significantly reduced the release of $120 \mathrm{~K}$ EVs by adult $F$. hepatica in vitro.

\section{GW4869 causes ultrastructural disruption of adult fluke tissues in vitro}

Fluke tissue sections were viewed by TEM to observe the effects of GW4869 treatment at the ultrastructural level. Whilst the tegument of control F. hepatica appeared normal (Fig. 8A), the tegumental syncytium of GW4869-treated flukes was severely vacuolated, although no blebbing of the apical plasma membrane (a common feature of drug-induced damage in trematodes) was apparent (Fig. 8B). Furthermore, whilst fewer T2 secretory bodies were visible, the distribution and number of $\mathrm{T} 1$ secretory bodies appeared similar to the controls and the spines appeared normal (Fig. 8B). In comparison to controls (Fig. 8C), the gastrodermal cells of GW4869-treated flukes appeared 
to be undergoing higher levels of autophagy, as evidenced by the abundance of autophagosomes (Fig. 8D). Some vacuolation of the gastrodermal cell cytoplasm was visible following GW4869 treatment (Fig. 8D), whilst the lamellae appeared normal (Fig. 8D). TEM also revealed that, compared to the controls (Fig. 8E), the parenchymal tissue of GW4869-treated F. hepatica appeared highly disorganised with numerous large electron-lucent vacuoles dominating the tissue (Fig. 8F). Additionally, compared to the controls (Fig. 8G), more vacuolation was evident in the protonephridial duct epithelia of GW4869-treated F. hepatica (Fig. 8H), although the lipid droplets within the ducts appeared normal.

\section{Discussion}

Helminth EVs (hEVs) are emerging as important mediators of the host-parasite interaction and are consequently being investigated as novel targets for parasite control (Wu et al., 2018). These studies have so far focussed on preventing the internalisation of hEVs by host cells (Chaiyadet et al., 2015; de la Torre-Escudero et al., 2019) and on the potential of hEVs as vaccine candidates (Kifle et al., 2017). However, the effect of blocking hEV release on parasite survival within the host has yet to be investigated due to our limited understanding of the molecular machinery that drives $\mathrm{hEV}$ biogenesis and secretion. Accordingly, this work aimed to develop our knowledge of the mechanisms underlying $\mathrm{hEV}$ formation by firstly investigating the sites of $\mathrm{EV}$ secretion by $F$. hepatica and secondly attempting to disrupt FhEV release by targeting putative EV biogenesis molecules.

FhEVs can be separated into two distinct (albeit crude) populations by differential centrifugation at $15,000 \times g(15 \mathrm{~K}$ EVs) and $120,000 \times g(120 \mathrm{~K}$ EVs $)$ which were originally proposed to originate from the gut and the tegument, respectively (Cwiklinski et al., 2015; de la Torre-Escudero et al., 2019; Marcilla et al., 2012). However, our immunolocalisation data suggest that FhEVs are derived from multiple cell types. The localisation of several FhEV marker proteins to vesicular structures within the parenchyma indicates that these cells play a previously unknown 
role in EV biogenesis. Immunolabelling of parenchymal cells resembling the PC2-type described in F. gigantica (Pankao et al., 2006) suggested that these cells have a secretory function in addition to their proposed roles in lipid storage and metabolism. For the parenchymal cell-derived vesicles to be secreted extra-corporeally, they would have to be trafficked to suitable sites within the fluke that are permissive to their release. In this regard, vesicles bearing the same markers were also seen outside the parenchymal cells (around their periphery or in the interstitial material) and in close proximity to the gut, tegument and protonephridial ducts, indicating that they are trafficked within the parenchyma to these potential sites of release. Moreover, similar structures were visible in rear extensions of gastrodermal cells that protruded into the parenchymal tissue, suggesting that there may be a transition of these vesicles between the parenchyma and the basolateral membrane of gastrodermal cells. A possible mechanism for EV transfer between cells is adsorptive-mediated transcytosis which enables EVs released by erythrocytes to cross the blood-brain barrier (Matsumoto et al., 2017). Such a process in gastrodermal cells could be mediated by Rab11 (enriched on the surface of $120 \mathrm{~K}$ EVs; de la Torre-Escudero et al., 2019) which mediates transcytosis of vesicles across canine kidney cells (Casanova et al., 1999; Wang et al., 2000), although this mechanism requires experimental investigation.

As had been proposed by Cwiklinski et al. (2015), the gut was found to be the dominant site of $15 \mathrm{~K} \mathrm{EV}$ release. In particular, those EVs immunopositive for tyrosinated $\alpha$-tubulin, FhSMPDL $3^{159}$, FhCL1 and FhCD63rec were shown by oral sucker ligation to originate from the gastrointestinal tract. Unexpectedly, oral sucker ligation also demonstrated that the majority of 120K EVs originate from the gut. Components of both the ESCRT-dependent (FhALIX) and ESCRT-independent (SMases) EV biogenesis pathways were identified in the gastrodermal cells and the varied localisation of FhEV marker proteins indicates that the gut is capable of secreting multiple sub-populations of EVs. Whilst the gastrodermal cells may just act as a conduit for release of vesicles trafficked from the parenchyma, we have previously detected FhRal-A in F. hepatica gastrodermal cells (de la Torre-Escudero et al., 2019) which is thought to initiate a pathway 
involving ALIX and syntenin that results in the budding of ILVs (Hyenne et al., 2016). However, co-localisation and functional studies would be required to verify this mechanism.

The immunolocalisation studies and in vitro ligation experiments showed that the tegument could also contribute to $15 \mathrm{~K}$ and $120 \mathrm{~K}$ EV release, although to a lesser extent than the gut. Surprisingly, DL did not affect $15 \mathrm{~K}$ EV total protein or particle concentration. However, the DL $15 \mathrm{~K} \mathrm{EV}$ population did have a markedly different protein profile (with a significant reduction in gut-derived proteins), indicating that some of the material was shed from the tegument as a result of ligation which masked the true effect of DL on $15 \mathrm{~K}$ EV total protein and particle concentration. Notably, there was no increase in the protein content of the DL $15 \mathrm{~K} \mathrm{EVs}$, suggesting the shedding of membranous material (which pelleted at 15,000 x $g$ ) rather than protein aggregates. Therefore, DL may have induced the release of blebs which pinch directly from the apical plasma membrane (APM) of the tegumental syncytium (de la Torre-Escudero et al., 2016). This supports the proposal that external stress (in this case, DL) to helminths causes the release of blebs as a mechanism of tegument repair. A small number of $120 \mathrm{~K}$ EVs were still released by $F$. hepatica following DL. Whilst these could be leakage from the gut and the protonephridial system due to incomplete ligation, it could also indicate that some $120 \mathrm{~K}$ EVs originate from the tegument. TEM studies have observed MVB-like structures releasing small $(<50 \mathrm{~nm})$ EVs at the tegument APM (de la TorreEscudero et al., 2016; Wilson et al., 2011) and FhTeg1, which was identified in $120 \mathrm{~K}$ EVs by mass spectrometry (Cwiklinski et al. 2015), showed this distribution pattern. As FhTeg1 immunolabelling was not observed at any other secretory interface, the tegument is likely to be the source of FhTeg1-positive 120K EVs.

Compared to the gut and the tegument, the role of the protonephridial system in the hostparasite interaction has been somewhat overlooked. During infection, the protonephridial system has been associated with the excretion of lipids and anthelmintics (Burren et al., 1967; Kusel et al., 2009; Wilson and Webster, 1974), but our current data suggests an additional role in the release of FhEVs. The in vitro ligation experiment indicated that a FhRal-A-positive sub-population of 120K 
EVs are released from the excretory pore and the localisation of several EV biogenesis proteins (FhALIX, FhTSG101, FhRal-A and FhSMPD2) to the protonephridial duct epithelium suggests that $120 \mathrm{~K}$ EVs may also be generated (or at least transported) by this organ system. Recently, the excretory system has also been suggested as a source of EVs from the nematodes Ascaris suum and Brugia malayi (Hansen et al., 2019; Harischandra et al., 2018). Interestingly, ALIX was localised to the excretory duct of B. malayi (Harischandra et al., 2018) which could indicate a conserved role of this protein in the formation of EVs in the excretory ducts of helminths. Notably, EV biogenesis proteins did not localise to lipid droplets in the protonephridia of $F$. hepatica, nor did SMase inhibition impact lipid droplet formation (see below). Whilst the molecular mechanism responsible for lipid droplet formation in helminths remains unresolved, the initial characterisation of the lipid droplets released from the excretory pore of $F$. hepatica suggested that they have a complex composition and function in the removal of lipid (or lipid-soluble) metabolic waste products from parasite tissues (Burren et al., 1967; Moss, 1970). The effects of excreting such vast quantities of lipid (10\% of the fluke's total lipid content during $4 \mathrm{~h}$ in vitro culture; Burren et al., 1967) on host cell physiology are unknown. However, the recent description of the lipase-independent release of lipid-filled vesicles from mouse adipocytes and their effects on macrophage differentiation and function (Flaherty et al., 2019) sets an interesting precedent for the possible roles of such bioactive lipids, or proteins embedded in the lipid droplets' lamellar coat (Bennett, 1977), excreted during helminth infection.

BLAST surveys indicated that $F$. hepatica has a distinct SMase repertoire to humans and phylogenetic analysis showed that fluke acid-like SMases clustered separately to their human counterparts. Furthermore, key functional residues were not conserved, including $N$-glycosylation sites essential for HsSMPDL3a nucleotide phosphodiesterase activity (Traini et al., 2017) which indicates that the fluke acid-like enzymes have distinct functions or catalytic mechanisms. Whilst HsSMPDL3a does not have SMase activity (Traini et al., 2014), HsSMPDL3b is thought to have SMase activity when tethered to a lipid bilayer (Gorelik et al., 2016a). Conceivably, FhSMPDL3 ${ }^{138}$ 
could be tethered to lipid bilayers by its predicted transmembrane region and proteomic analysis has shown that FhSMPDL $3^{159}$ and FhSMPDL $3^{138}$ are associated with FhEV membranes (Cwiklinski et al., 2015; de la Torre-Escudero et al., 2019), whilst FhSMPDL3 ${ }^{159}$ was localised to the periphery of cells. This localisation pattern of FhSMPDL3 ${ }^{159}$ and FhSMPDL $3^{138}$ could therefore permit SMase activity, but direct biochemical characterisation of these proteins would be required to confirm this.

Functionally important residues and motifs were, however, conserved in FhSMPD2, including the DK switch which acts in both catalysis and interactions with the neutral SMase inhibitor GW4869 (Airola et al., 2017). Indeed, treating FhEVs with GW4869 inhibited FhEVassociated neutral SMase activity in a dose-dependent manner. However, residual activity following GW4869 treatment suggests that FhSMPD2 may be refractory to this inhibitor (compared to human enzymes) as has been reported for insect SMase (Zhou et al. 2017).

Treating adult $F$. hepatica with GW4869 in vitro had a minor impact on $15 \mathrm{~K}$ EV release which points to a SMase-independent mode of biogenesis for these vesicles in F. hepatica. In contrast, GW4869 caused a major reduction in the release of $120 \mathrm{~K}$ EVs which reflects the impact of this molecule on exosome/small EV release reported in the literature (Essandoh et al., 2015; Li et al., 2013; Menck et al., 2017; Willms et al., 2016) and suggests that 120K EVs form in a ceramidedependent manner. Some 120K EVs, however, were still released following GW4869 treatment which indicates either that GW4869 does not completely inhibit FhSMase activity (as was demonstrated in the assays using isolated EVs) or that hybrid/multiple EV biogenesis pathways exist in F. hepatica; a proposition supported by the enrichment of ESCRT proteins in the 120K EVs in particular (Cwiklinski et al., 2015; de la Torre-Escudero et al., 2019).

GW4869-treated fluke tissue was examined by microscopy to provide insight into its mechanism of action. Whilst increased autophagy indicated that there was some disruption to the gut, more severe disruption was observed in the parenchymal tissue of GW4869-treated flukes, including in sub-gastrodermal and sub-protonephridial regions. This further supports the differential origins of 15K EVs and 120K EVs bearing the same markers (Cwiklinski et al., 2015; de la Torre- 
Escudero et al., 2019). For example, GW4869 treatment had no effect on the release of FhCD63recpositive $15 \mathrm{~K}$ EVs (secreted directly by gastrodermal cells) but had a significant impact on the release of FhCD63rec-positive $120 \mathrm{~K} \mathrm{EVs} \mathrm{(which} \mathrm{may} \mathrm{be} \mathrm{synthesised} \mathrm{in} \mathrm{the} \mathrm{parenchymal} \mathrm{cells}$ before being trafficked to the gastrodermal cells for secretion). The extensive vacuolation of fluke tissue may be attributed to the inhibition of sphingomyelin hydrolysis by GW4869. Indeed, the ultrastructural effects of GW4869 were broadly similar to those seen in sphingomyelin storage diseases such as Niemann-Pick disease where sphingomyelin accumulates in the cell (Elleder, 1989). Whilst GW4869 was non-lethal in vitro, it remains to be determined whether the inhibition of FhEV release seen here would be sufficient to impair parasite survival in vivo. However, ivermectin-induced suppression of EV release from B. malayi has been proposed to enable immunemediated parasite killing in vivo (Harischandra et al., 2018).

This study has shown that EV biogenesis in F. hepatica is more complex than first thought, with the parasite capable of secreting a range of FhEV subtypes from a variety of tissues/organ systems and via multiple biogenesis pathways. However, despite the complexities of FhEV biogenesis identified in this study, it was revealed for the first time that targeted disruption of EV biogenesis and release in helminths is possible. In doing so, we provide proof-of-concept for future in vivo studies investigating EV secretion as a target for parasite control.

\section{Acknowledgements}

This work was supported by a grant to M.W.R. (BB/L019612/1) from the Biotechnology and Biological Sciences Research Council (BBSRC). A.P.S.B. was supported by a postgraduate studentship from the Department for the Economy (DfE) Northern Ireland. We thank Deborah Moffett of the Veterinary Sciences Division, Agri-Food and Biosciences Institute (AFBI), Belfast for assistance with transmission electron microscopy and Associate Professor Lidia Tajber, Trinity College Dublin, for access to the NanoSight NS300. 


\section{References}

Airola, M. V, Shanbhogue, P., Shamseddine, A.A., Guja, K.E., Senkal, C.E., Maini, R., Bartke, N., Wu, B.X., Obeid, L.M., Garcia-Diaz, M., Hannun, Y.A., 2017. Structure of human nSMase2 reveals an interdomain allosteric activation mechanism for ceramide generation. Proc. Natl. Acad. Sci. U. S. A. 114, E5549-E5558.

Bennett, C.E., 1977. Fasciola hepatica: development of excretory and parenchymal systems during migration in the mouse. Exp. Parasitol. 41, 43-53.

Burren, C.H., Ehrlich, I., Johnson, P., 1967. Excretion of lipids by the liver fluke (Fasciola hepatica L). Lipids 2, 353-356.

Casanova, J.E., Wang, X., Kumar, R., Bhartur, S.G., Navarre, J., Woodrum, J.E., Altschuler, Y., Ray, G.S., Goldenring, J.R., 1999. Association of Rab25 and Rab11a with the apical recycling system of polarized Madin-Darby canine kidney cells. Mol. Biol. Cell 10, 47-61.

Chaiyadet, S., Sotillo, J., Smout, M., Cantacessi, C., Jones, M.K., Johnson, M.S., Turnbull, L., Whitchurch, C.B., Potriquet, J., Laohaviroj, M., Mulvenna, J., Brindley, P.J., Bethony, J.M., Laha, T., Sripa, B., Loukas, A., 2015. Carcinogenic liver fluke secretes extracellular vesicles that promote cholangiocytes to adopt a tumorigenic phenotype. J. Infect. Dis. 212, 1636-45.

Christ, L., Raiborg, C., Wenzel, E.M., Campsteijn, C., Stenmark, H., 2017. Cellular functions and molecular mechanisms of the ESCRT membrane-scission machinery. Trends Biochem. Sci. $42,42-56$.

Cwiklinski, K., de la Torre-Escudero, E., Trelis, M., Bernal, D., Dufresne, P.J., Brennan, G.P., O’Neill, S., Tort, J., Paterson, S., Marcilla, A., Dalton, J.P., Robinson, M.W., 2015. The extracellular vesicles of the helminth pathogen, Fasciola hepatica: biogenesis pathways and cargo molecules involved in parasite pathogenesis. Mol. Cell. Proteomics 14, 3258-3273.

Dalton, J.P., Robinson, M.W., Mulcahy, G., O’Neill, S.M., Donnelly, S., 2013. Immunomodulatory 
molecules of Fasciola hepatica: candidates for both vaccine and immunotherapeutic development. Vet. Parasitol. 195, 272-85.

de la Torre-Escudero, E., Bennett, A.P.S., Clarke, A., Brennan, G.P., Robinson, M.W., 2016. Extracellular vesicle biogenesis in helminths: more than one route to the surface? Trends Parasitol. 32, 921-929.

de la Torre-Escudero, E., Gerlach, J.Q., Bennett, A.P.S., Cwiklinski, K., Jewhurst, H.L., Huson, K.M., Joshi, L., Kilcoyne, M., O’Neill, S., Dalton, J.P., Robinson, M.W., 2019. Surface molecules of extracellular vesicles secreted by the helminth pathogen Fasciola hepatica direct their internalisation by host cells. PLoS Negl. Trop. Dis. 13, e0007087.

Di Maggio, L.S., Tirloni, L., Pinto, A.F.M., Diedrich, J.K., Yates Iii, J.R., Benavides, U., Carmona, C., da Silva Vaz, I., Berasain, P., 2016. Across intra-mammalian stages of the liver fluke Fasciola hepatica: a proteomic study. Sci. Rep. 6, 32796.

El Andaloussi, S., Mäger, I., Breakefield, X.O., Wood, M.J.A., 2013. Extracellular vesicles: biology and emerging therapeutic opportunities. Nat. Rev. Drug Discov. 12, 347-57.

Elleder, M., 1989. Niemann-Pick disease. Pathol. Res. Pract. 185, 293-328.

Essandoh, K., Yang, L., Wang, X., Huang, W., Qin, D., Hao, J., Wang, Y., Zingarelli, B., Peng, T., Fan, G.C., 2015. Blockade of exosome generation with GW4869 dampens the sepsis-induced inflammation and cardiac dysfunction. Biochim. Biophys. Acta - Mol. Basis Dis. 1852, 23622371.

Felsenstein, J., 1985. Confidence limits on phylogenies: an approach using the bootstrap. Evolution (N. Y). 39, 783-791.

Flaherty, S.E., Grijalva, A., Xu, X., Ables, E., Nomani, A., Ferrante, A.W., 2019. A lipaseindependent pathway of lipid release and immune modulation by adipocytes. Science 363, 989-993. 
Gorelik, A., Heinz, L.X., Illes, K., Superti-Furga, G., Nagar, B., 2016a. Crystal structure of the acid sphingomyelinase-like phosphodiesterase SMPDL3B provides insights into determinants of substrate specificity. J. Biol. Chem. 291, 24054-24064.

Gorelik, A., Illes, K., Heinz, L.X., Superti-Furga, G., Nagar, B., 2016b. Crystal structure of mammalian acid sphingomyelinase. Nat. Commun. 7, 1-9.

Gradilla, A.C., González, E., Seijo, I., Andrés, G., Bischoff, M., González-Mendez, L., Sánchez, V., Callejo, A., Ibáñez, C., Guerra, M., Ortigão-Farias, J.R., Sutherland, J.D., González, M., Barrio, R., Falcón-Pérez, J.M., Guerrero, I., 2014. Exosomes as Hedgehog carriers in cytoneme-mediated transport and secretion. Nat. Commun. 5, 5649.

Hanna, R.E.B., 1980. Fasciola hepatica: autoradiography of protein synthesis, transport, and secretion by the tegument. Exp. Parasitol. 50, 297-304.

Hansen, E.P., Fromm, B., Andersen, S.D., Marcilla, A., Andersen, K.L., Borup, A., Williams, A.R., Jex, A.R., Gasser, R.B., Young, N.D., Hall, R.S., Stensballe, A., Ovchinnikov, V., Yan, Y., Fredholm, M., Thamsborg, S.M., Nejsum, P., 2019. Exploration of extracellular vesicles from Ascaris suum provides evidence of parasite-host cross talk. J. Extracell. vesicles 8, 1578116.

Harischandra, H., Yuan, W., Loghry, H.J., Zamanian, M., Kimber, M.J., 2018. Profiling extracellular vesicle release by the filarial nematode Brugia malayi reveals sex-specific differences in cargo and a sensitivity to ivermectin. PLoS Negl. Trop. Dis. 12, e0006438.

Heinz, L.X., Baumann, C.L., Köberlin, M.S., Snijder, B., Gawish, R., Shui, G., Sharif, O., Aspalter, I.M., Müller, A.C., Kandasamy, R.K., Breitwieser, F.P., Pichlmair, A., Bruckner, M., Rebsamen, M., Blüml, S., Karonitsch, T., Fauster, A., Colinge, J., Bennett, K.L., Knapp, S., Wenk, M.R., Superti-Furga, G., 2015. The lipid-modifying enzyme SMPDL3B negatively regulates innate immunity. Cell Rep. 11, 1919-28.

Hyenne, V., Labouesse, M., Goetz, J.G., 2016. The Small GTPase Ral orchestrates MVB 
667

668

669

670

671

672

673

674

675

676

677

678

679

680

681

682

683

Kifle, D.W., Sotillo, J., Pearson, M.S., Loukas, A., 2017. Extracellular vesicles as a target for the development of anti-helminth vaccines. Emerg. Top. Life Sci. 1, 659-665.

Kowal, J., Tkach, M., Théry, C., 2014. Biogenesis and secretion of exosomes. Curr. Opin. Cell Biol. 29, 116-125.

Kusel, J.R., McVeigh, P., Thornhill, J.A., 2009. The schistosome excretory system: a key to regulation of metabolism, drug excretion and host interaction. Trends Parasitol. 25, 353-358.

Li, J., Liu, K., Liu, Y., Xu, Y., Zhang, F., Yang, H., Liu, J., Pan, T., Chen, J., Wu, M., Zhou, X., Yuan, Z., 2013. Exosomes mediate the cell-to-cell transmission of IFN- $\alpha$-induced antiviral activity. Nat. Immunol. 14, 793-803.

Marcilla, A., Trelis, M., Cortés, A., Sotillo, J., Cantalapiedra, F., Minguez, M.T., Valero, M.L., Sánchez del Pino, M.M., Muñoz-Antoli, C., Toledo, R., Bernal, D., 2012. Extracellular vesicles from parasitic helminths contain specific excretory/secretory proteins and are internalized in intestinal host cells. PLoS One 7, e45974.

Matsumoto, J., Stewart, T., Sheng, L., Li, N., Bullock, K., Song, N., Shi, M., Banks, W.A., Zhang, J., 2017. Transmission of $\alpha$-synuclein-containing erythrocyte-derived extracellular vesicles across the blood-brain barrier via adsorptive mediated transcytosis: another mechanism for initiation and progression of Parkinson's disease? Acta Neuropathol. Commun. 5, 71.

Menck, K., Sönmezer, C., Worst, T.S., Schulz, M., Dihazi, G.H., Streit, F., Erdmann, G., Kling, S., Boutros, M., Binder, C., Gross, J.C., 2017. Neutral sphingomyelinases control extracellular vesicles budding from the plasma membrane. J. Extracell. vesicles 6, 1378056.

Morphew, R.M., Wright, H.A., LaCourse, E.J., Woods, D.J., Brophy, P.M., 2007. Comparative proteomics of excretory-secretory proteins released by the liver fluke Fasciola hepatica in sheep host bile and during in vitro culture ex host. Mol. Cell. Proteomics 6, 963-72. 
Moss, G.D., 1970. The excretory metabolism of the endoparasitic digenean Fasciola hepatica and its relationship to its respiratory metabolism. Parasitology 60, 1-19.

Pankao, V., Sirisriro, A., Grams, R., Vichasri-Grams, S., Meepool, A., Kangwanrangsan, N., Wanichanon, C., Ardseungneon, P., Viyanant, V., Upatham, E.S., Sobhon, P., 2006. Classification of the parenchymal cells in Fasciola gigantica based on ultrastructure and their expression of fatty acid binding proteins (FABPs). Vet. Parasitol. 142, 281-292.

Robinson, M.W., Menon, R., Donnelly, S.M., Dalton, J.P., Ranganathan, S., 2009. An integrated transcriptomics and proteomics analysis of the secretome of the helminth pathogen Fasciola hepatica: proteins associated with invasion and infection of the mammalian host. Mol. Cell. Proteomics 8, 1891-907.

Robinson, M.W., Trudgett, A., Hoey, E.M., Fairweather, I., 2002. Triclabendazole-resistant Fasciola hepatica: $\beta$-tubulin and response to in vitro treatment with triclabendazole. Parasitology 124, 325-38.

Rohrbacher, G.H., 1957. Observations of the survival in vitro of bacteria-free adult common liver flukes, Fasciola hepatica Linn., 1758. J. Parasitol. 43, 9-18.

Roig, J., Saiz, M.L., Galiano, A., Trelis, M., Cantalapiedra, F., Monteagudo, C., Giner, E., Giner, R.M., Recio, M.C., Bernal, D., Sánchez-Madrid, F., Marcilla, A., 2018. Extracellular vesicles from the helminth Fasciola hepatica prevent DSS-induced acute ulcerative colitis in a Tlymphocyte independent mode. Front. Microbiol. 9, 1036.

Saitou, N., Nei, M., 1987. The neighbor-joining method: a new method for reconstructing phylogenetic trees. Mol. Biol. Evol. 4, 406-25.

Tamura, K., Stecher, G., Peterson, D., Filipski, A., Kumar, S., 2013. MEGA6: Molecular Evolutionary Genetics Analysis version 6.0. Mol. Biol. Evol. 30, 2725-9.

Threadgold, L.T., 1963. The tegument and associated structures of Fasciola hepatica. Quart. J. 
Tomiuk, S., Zumbansen, M., Stoffel, W., 2000. Characterization and subcellular localization of murine and human magnesium-dependent neutral sphingomyelinase. J. Biol. Chem. 275, $5710-5717$.

Traini, M., Kumaran, R., Thaysen-Andersen, M., Kockx, M., Jessup, W., Kritharides, L., 2017. Nglycosylation of human sphingomyelin phosphodiesterase acid-like 3A (SMPDL3A) is essential for stability, secretion and activity. Biochem. J. 474, 1071-1092.

Traini, M., Quinn, C.M., Sandoval, C., Johansson, E., Schroder, K., Kockx, M., Meikle, P.J., Jessup, W., Kritharides, L., 2014. Sphingomyelin phosphodiesterase acid-like 3A (SMPDL3A) is a novel nucleotide phosphodiesterase regulated by cholesterol in human macrophages. J. Biol. Chem. 289, 32895-32913.

Trajkovic, K., Hsu, C., Chiantia, S., Rajendran, L., Wenzel, D., Wieland, F., Schwille, P., Brügger, B., Simons, M., 2008. Ceramide triggers budding of exosome vesicles into multivesicular endosomes. Science 319, 1244-1247.

Truman, J.P., Al Gadban, M.M., Smith, K.J., Jenkins, R.W., Mayroo, N., Virella, G., Lopes-Virella, M.F., Bielawska, A., Hannun, Y.A., Hammad, S.M., 2012. Differential regulation of acid sphingomyelinase in macrophages stimulated with oxidized low-density lipoprotein (LDL) and oxidized LDL immune complexes: role in phagocytosis and cytokine release. Immunology $136,30-45$.

van Niel, G., D’Angelo, G., Raposo, G., 2018. Shedding light on the cell biology of extracellular vesicles. Nat. Rev. Mol. Cell Biol. 19, 213-228.

Verderio, C., Gabrielli, M., Giussani, P., 2018. Role of sphingolipids in the biogenesis and biological activity of extracellular vesicles. J. Lipid Res. 59, 1325-1340.

Wang, X., Kumar, R., Navarre, J., Casanova, J.E., Goldenring, J.R., 2000. Regulation of vesicle 
trafficking in Madin-Darby canine kidney cells by Rab11a and Rab25. J. Biol. Chem. 275, 29138-46.

Willms, E., Johansson, H.J., Mäger, I., Lee, Y., Blomberg, K.E.M., Sadik, M., Alaarg, A., Smith, C.I.E., Lehtiö, J., EL Andaloussi, S., Wood, M.J.A., Vader, P., 2016. Cells release subpopulations of exosomes with distinct molecular and biological properties. Sci. Rep. 6, 22519.

Wilson, R.A., Webster, L.A., 1974. Protonephridia. Biol. Rev. 49, 127-160.

Wilson, R.A., Wright, J.M., de Castro-Borges, W., Parker-Manuel, S.J., Dowle, A.A., Ashton, P.D., Young, N.D., Gasser, R.B., Spithill, T.W., 2011. Exploring the Fasciola hepatica tegument proteome. Int. J. Parasitol. 41, 1347-1359.

Wu, Zhenyu, Wang, Lingling, Li, J., Wang, Lifu, Wu, Zhongdao, Sun, X., 2018. Extracellular vesicle-mediated communication within host-parasite interactions. Front. Immunol. 9, 3066.

Zakeri, A., Hansen, E.P., Andersen, S.D., Williams, A.R., Nejsum, P., 2018. Immunomodulation by helminths: intracellular pathways and extracellular vesicles. Front. Immunol. 9.

Zhou, Y., Lin, X.-W., Begum, M.-A., Zhang, C.-H., Shi, X.-X., Jiao, W.-J., Zhang, Y.-R., Yuan, J.Q., Li, H.-Y., Yang, Q., Mao, C., Zhu, Z.-R., 2017. Identification and characterization of Laodelphax striatellus (Insecta: Hemiptera: Delphacidae) neutral sphingomyelinase. Insect Mol. Biol. 26, 392-402.

Zuckerandl, E., Pauling, L., 1965. Evolutionary divergence and convergence in proteins, in: Bryson, V., Vogel, H.J. (Eds.), Evolving Genes and Proteins. Academic Press, New York, pp. 97-166.

\section{Legends to Figures}


Fig. 1. Proteins enriched in $F$. hepatica EVs localise to the gastrodermal cells in adult $F$. hepatica tissue sections. (A) Toluidine blue staining showing the architecture of the gastrodermal tract. (B) Pre-immune serum showing no specific immunofluorescence in the gut. (C-H) Immunostaining (green, or red in $\mathrm{H}$ ) patterns in the gut show puncta of fluorescence concentrated at the apices of the gastrodermal cells (arrowheads in C, FhALIX; F, FhDM9; G, FhTIP; and H, tyrosinated $\alpha$-tubulin); diffuse FhSMPD2-positive immunofluorescence (D); low intensity staining of the lamellae (D, FhSMPD2; E, FhSMPDL $3^{159}$ ); and puncta of fluorescence in the sub-gastrodermal region (E, FhSMPDL3 ${ }^{159}$; F, FhDM9; G, FhTIP). FhDM9 is also present in large vesicular structures in the gut lumen (arrow, F). Panels B, D, and E display DAPI nuclear staining (blue). G, gut; L, lumen. Scale bars: $25 \mu \mathrm{m}(\mathrm{A}), 7.5 \mu \mathrm{m}(\mathrm{B}-\mathrm{H})$.

Fig. 2. Immunostaining (green) of proteins enriched in F. hepatica EVs in the tegument and subtegumental regions of adult $F$. hepatica tissue sections. (A) Toluidine blue staining showing the architecture of the tegumental region. (B) Pre-immune serum showing no specific immunofluorescence. (C) The anti-FhSMPDL $3^{159}$ antibody labels the cytoplasmic connections (arrows) and basal part of the tegumental syncytium. (D-E) Immunoreactivity against FhDM9 (D) and FhTeg1 (E) is present in the tegumental apical plasma membrane, syncytium, and subtegmental areas (arrowheads). APM, apical plasma membrane; PN, protonephridial duct; S, spine; T, tegumental syncytium. Panels B, C, and E display DAPI nuclear staining (blue). Scale bars: 25 $\mu \mathrm{m}(\mathrm{A}), 7.5 \mu \mathrm{m}(\mathrm{B}-\mathrm{E})$.

\section{Fig. 3. Immunolocalisation of proteins enriched in F. hepatica EVs (green) to the protonephridial} ducts (PN) and associated parenchymal cells in adult $F$. hepatica tissue sections. (A) Toluidine blue staining showing a protonephridial duct embedded in the parenchymal tissue. (B) Pre-immune serum showing no specific immunofluorescence. (C-G) The lining of the protonephridial ducts 
display immunofluorescence positive for FhTSG101 (arrows, C), FhALIX (D), FhRal-A (E) and FhSMPD2 (F), whilst FhDM9 immunoreactivity is localised to the perinuclear region (arrow, G). Clusters of vesicular structures (arrowheads) are also visible in parenchymal cells, including those adjacent to the protonephridial ducts. Panels B, C, E, and F display DAPI nuclear staining (blue). Scale bars: $7.5 \mu \mathrm{m}(\mathrm{A}-\mathrm{B}, \mathrm{D}-\mathrm{G}), 25 \mu \mathrm{m}(\mathrm{C})$.

Fig. 4. Parenchymal cells containing vesicular structures immunopositive for proteins enriched in $F$. hepatica EVs (green, top row) and the same cells stained with toluidine blue (bottom row). Panel (B) has DAPI nuclear staining (blue). Arrowheads indicate immunopositive clusters of vesicular structures within parenchymal cells. (A) FhTSG101. (B) FhSMPD2. (C) FhDM9. (D-F) The subgastrodermal parenchymal cells labelled by these antibodies have a slightly basophilic cytoplasm and contain many vesicular structures which are highly basophilic. This cell type resembles the PC2 parenchymal cells characterised by Pankao et al. (2006). G, gut; L, gut lumen; PN, protonephridia. Scale bars, $7.5 \mu \mathrm{m}$.

Fig. 5. Representative images of ligated adult $F$. hepatica after $3 \mathrm{~h}$ in vitro culture (left-hand panels), and the resulting $15 \mathrm{~K} \mathrm{EV}$ pellet recovered from the culture media (right-hand panels). The dashed circle highlights the location of the pellet. (A) Non-ligated control. (B) Oral sucker ligated. (C) Excretory pore ligated. (D) Oral sucker and excretory pore ligated.

Fig. 6. The effect of oral sucker ligation (OSL; A-B), excretory pore ligation (EPL; C-D), and oral sucker and excretory pore ligation (double ligation, DL; E-F) on the release of F. hepatica $15 \mathrm{~K}$ (A, C, F) and $120 \mathrm{~K}$ (B, D, F) EVs as determined by Western blot against selected EV markers. Experiments were carried out in biological triplicate. Representative blots are shown in the lefthand panel and mean densitometric values expressed as a percentage of the controls $( \pm$ standard 
810 811 812 813

error) in the right-hand panel. *, $\mathrm{p}<0.05$; **, $\mathrm{p}<0.01$, *** $\mathrm{p}<0.001$, ****, $\mathrm{p}<0.0001$; ****** $\mathrm{p}$ $<0.000001$ (Student's t-test). Ctrl, control.

Fig. 7. (A) The effect of GW4869 on sphingomyelinase activity associated with 120K EVs. Bars represent percentage sphingomyelinase activity of EVs treated with either the vehicle control or increasing concentrations of GW4869. (B-D) The effect of GW4869 treatment on 120K EV release as determined by protein (B) and particle (C) concentration (expressed as a percentage of the controls) and Western blots against selected EV markers (D). Representative blots are shown in the left-hand panel and the right-hand panel displays mean densitometric values expressed as a percentage of the controls. Experiments were carried out in biological triplicate and mean values \pm standard error are shown. Ctrl, control. *, p < 0.05; **, p < 0.01; ***, p < 0.001; *****, p < 0.00001 (Student's t-test).

Fig. 8. Transmission electron micrographs showing the effect of GW4869 treatment on the ultrastructure of adult $F$. hepatica. (A) Control tegumental syncytium. (B) GW4869-treated tegumental syncytium displaying extensive vacuolation (asterisks). (C) Control gastrodermal cell. (D) GW4869-treated gastrodermal cell showing an accumulation of autophagosomes (arrows) and some vacuolation (asterisk). (E) Control parenchymal tissue. (F) The parenchymal tissue of GW4869-treated F. hepatica displays large, electron lucent vacuoles (asterisks). (G) Control protonephridial duct with lipid droplets (arrows). (H) Areas of vacuolation (arrowheads) are more evident in the protonephridial duct of GW4869-trated F. hepatica, whilst lipid droplets (arrows) are still present. PN, protonephridial duct; S, spine; V, vitelline follicle. Scale bars: $2 \mu \mathrm{m}(\mathrm{A}, \mathrm{C}), 5 \mu \mathrm{m}$ (B, E, H), $1 \mu \mathrm{m}(\mathrm{D}), 10 \mu \mathrm{m}(\mathrm{F}, \mathrm{G})$. 
Supplementary Fig. S1. The effect of oral sucker ligation (OSL; A-B), excretory pore ligation (EPL; C-D), and oral sucker and excretory pore ligation (double ligation, DL; E-F) on 15K and 120K EV protein $(\mathrm{A}, \mathrm{C}, \mathrm{E})$ and particle $(\mathrm{B}, \mathrm{D}, \mathrm{F})$ concentration (expressed as a percentage of the controls). Experiments were carried out in biological triplicate and mean values \pm standard error are shown. *, $\mathrm{p}<0.05 ; * *, \mathrm{p}<0.01$ (Student's t-test).

Supplementary Fig. S2. (A) A bootstrapped (1000 trials) neighbour-joining phylogenetic tree of human neutral (HsSMPD2 and HsSMPD3) and acid-like sphingomyelinase (SMase) family members (HsSMPD1, HsSMPDL3a and HsSMPDL3b) with F. hepatica orthologues (FhSMPD2, FhSMPDL $3^{159}$ and FhSMPDL ${ }^{138}$ ). Numbers next to nodes represent bootstrap values (given as percentages). Values over $65 \%$ are displayed. (B) Cartoon representation of domains in human neutral and acid-like sphingomyelinases in comparison to F. hepatica orthologues. Catalytic domains (EEP, endonuclease/exonuclease/phosphatase; MPP, metallo-phosphodiesterase) are in black, signal peptides (SP) in dark grey, and transmembrane regions (TM) in blue. Protein domain architectures were generated using InterPro (www.ebi.ac.uk/interpro; Mitchell et al., 2015).

Supplementary Fig. S3. (A) The effect of GW4869 on sphingomyelinase activity associated with 15K EVs. Bars represent percentage sphingomyelinase activity of EVs treated with either the vehicle control or increasing concentrations of GW4869. (B-D) The effect of GW4869 treatment on $15 \mathrm{~K}$ EV release as determined by protein (B) and particle (C) concentration (expressed as a percentage of the controls) and Western blots against selected EV markers (D). Representative blots are shown in the left-hand panel and the right-hand panel displays mean densitometric values expressed as a percentage of the controls. Experiments were carried out in biological triplicate and mean values \pm standard error are shown. Ctrl, control. *, $\mathrm{p}<0.05 ; * *, \mathrm{p}<0.01$ (Student's t-test). 
862 Supplementary Table 1: List of primary antibodies used for immunolocalisation and immunoblot analysis.

\begin{tabular}{|c|c|c|c|c|c|c|c|}
\hline \multirow[t]{2}{*}{ Target protein } & \multirow[t]{2}{*}{ Target identifier } & \multirow[t]{2}{*}{ Antibody } & \multirow[t]{2}{*}{ Target peptide } & \multirow[t]{2}{*}{ Host } & \multicolumn{2}{|c|}{ Working concentration/dilution } & \multirow[t]{2}{*}{ Source } \\
\hline & & & & & Immunolocalisation & Immunoblotting & \\
\hline \multicolumn{8}{|l|}{ Polyclonal peptide antibodies } \\
\hline FhTSG101 & BN1106_s410B000432 & TSG101_18 & KSYKYARDVTNDVKC & Rabbit & $10 \mu \mathrm{g} / \mathrm{ml}$ & $1 \mu \mathrm{g} / \mathrm{ml}$ & GenScript \\
\hline FhALIX & BN1106_s1871B000313 & ALIX_2 & CTSSKKRPAPDRPPP & Rabbit & $10 \mu \mathrm{g} / \mathrm{ml}$ & - & GenScript \\
\hline FhRal-A & BN1106_s637B000246 & RAL_28 & CNKIDLTQERTVPFE & Rabbit & $10 \mu \mathrm{g} / \mathrm{ml}$ & $1 \mu \mathrm{g} / \mathrm{ml}$ & GenScript \\
\hline FhSMPD2 & BN1106_s7135B000046 & NSM_65 & CMDESPSDPDTKASG & Rabbit & $10 \mu \mathrm{g} / \mathrm{ml}$ & - & GenScript \\
\hline FhSMPDL $3^{159}$ & BN1106_s1285B000159 & ASM_1_41 & IEKGNDEGYAENKPC & Rabbit & $5 \mu \mathrm{g} / \mathrm{ml}$ & $1 \mu \mathrm{g} / \mathrm{ml}$ & GenScript \\
\hline FhCD63 receptor & BN1106_s1657B000161 & Cd63_9 & CGAKFTHKDSAAGRT & Rabbit & - & $0.125 \mu \mathrm{g} / \mathrm{ml}$ & GenScript \\
\hline FhDM9-containing protein & BN1106_s5689B000026 & DM9_26 & CPSGRGDVPHNAVQG & Rabbit & $7.5 \mu \mathrm{g} / \mathrm{ml}$ & - & GenScript \\
\hline FhTIP & BN1106_s92B000559 & TiP_5 & CYGASDSKRGTFLKP & Rabbit & $7.5 \mu \mathrm{g} / \mathrm{ml}$ & - & GenScript \\
\hline FhTeg1 & Q9Y0H2- & Teg1_44 & CPKGDGDPKPEPKGD & Rabbit & $10 \mu \mathrm{g} / \mathrm{ml}$ & - & GenScript \\
\hline \multicolumn{8}{|l|}{ Commercial monoclonal } \\
\hline Tyrosinated $\alpha$-tubulin & - & TUB-1A2 & - & Mouse & $1: 100$ & $1: 2500$ & Sigma-Aldrich \\
\hline \multicolumn{8}{|l|}{ Anti-serum } \\
\hline FhCL1 & U62288 & FhCL1 & Recombinant $F$. hepatica cathepsin L1 & Sheep & - & $1: 15,000$ & $\begin{array}{l}\text { de la Torre-Escudero et } \\
\text { al. (2019) }\end{array}$ \\
\hline
\end{tabular}


Figure 1

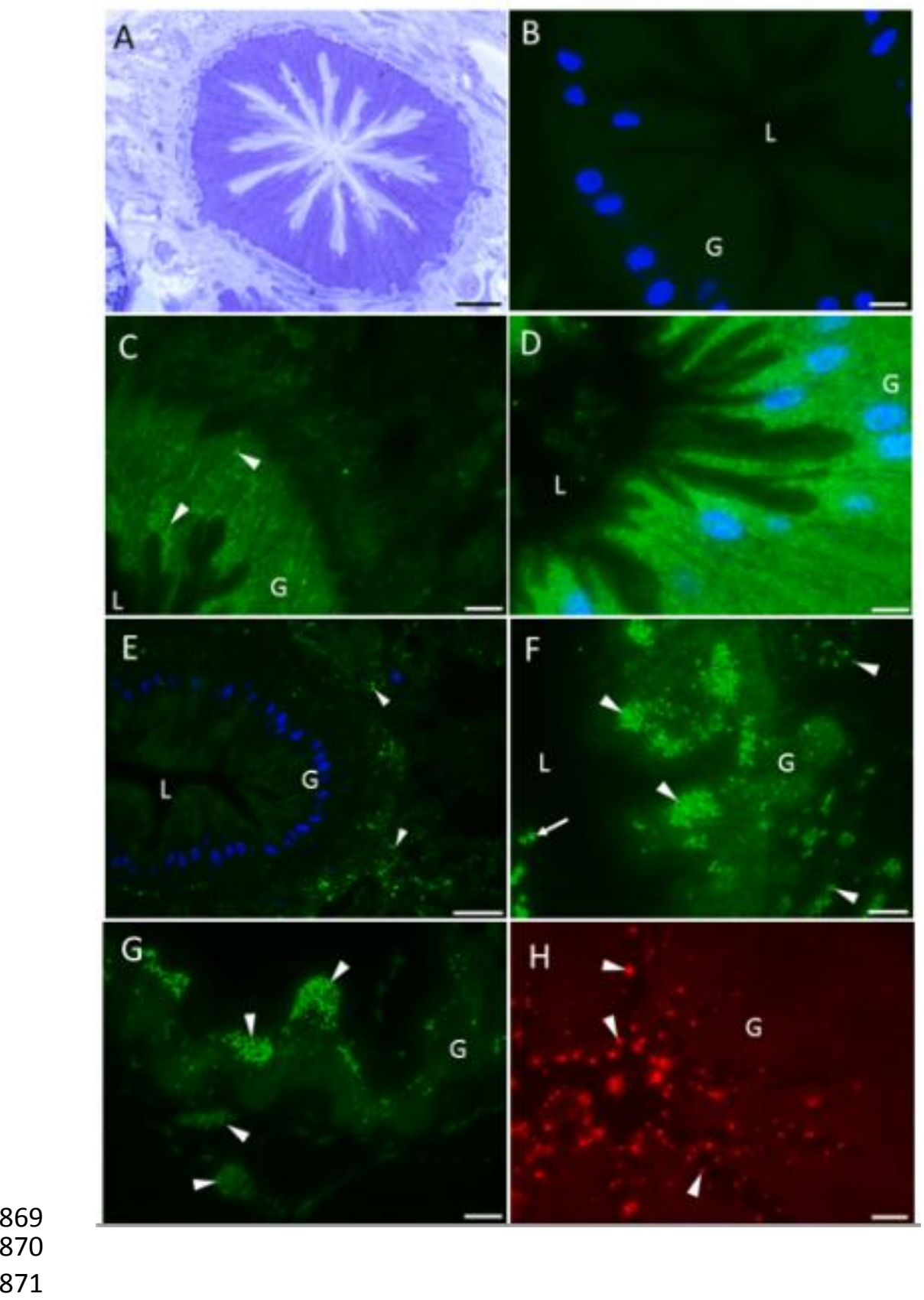


$872 \quad$ Figure 2

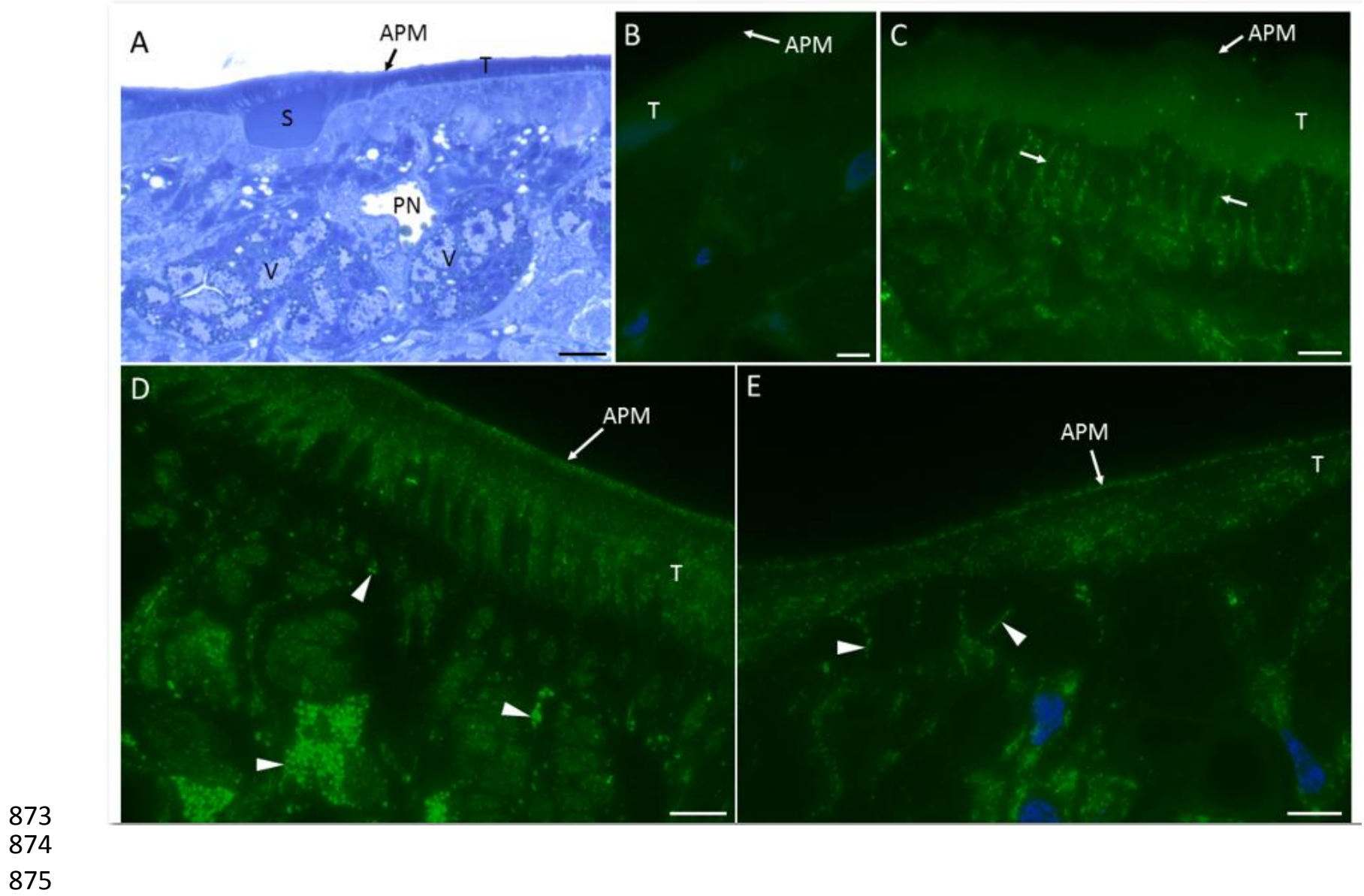




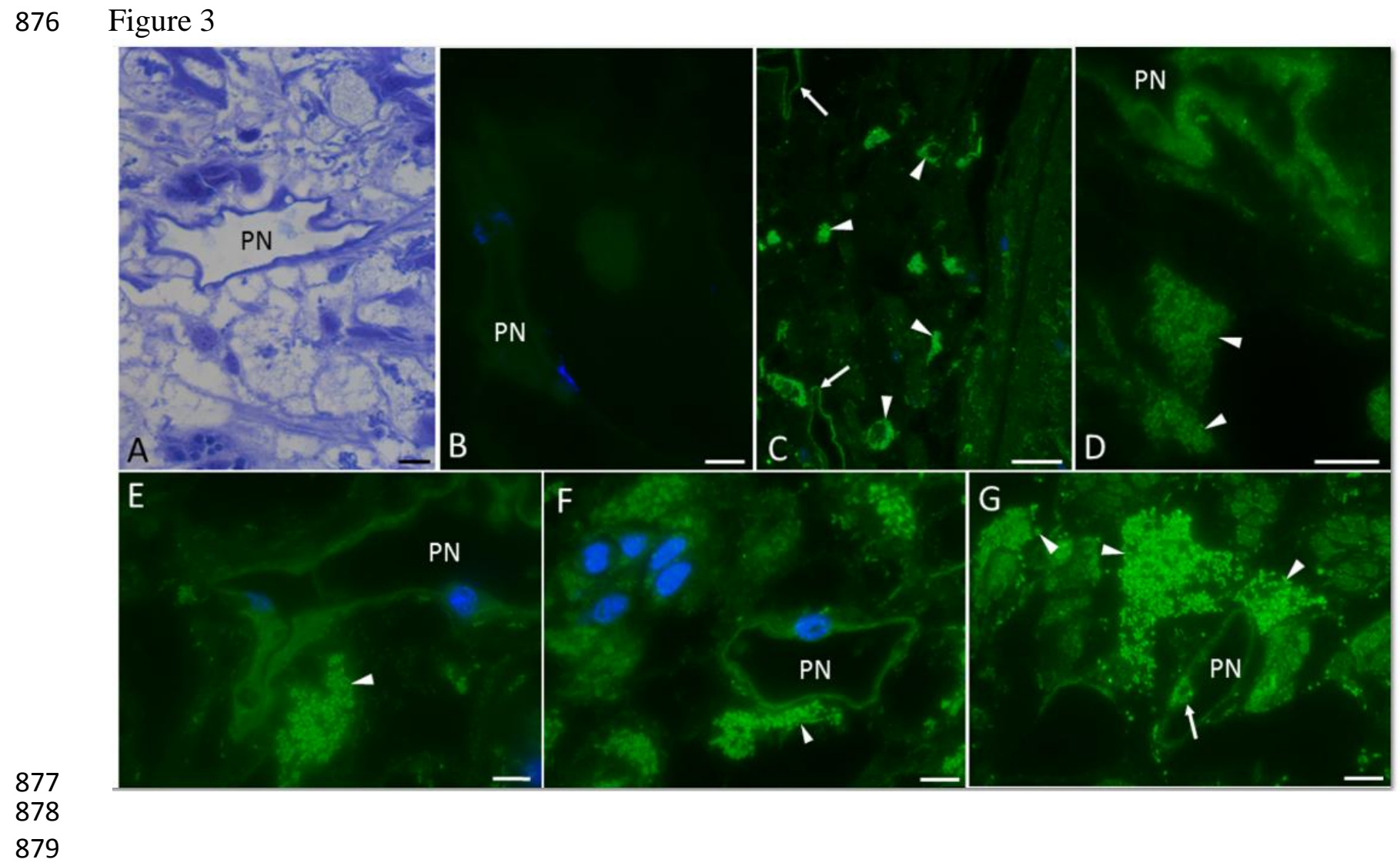


$880 \quad$ Figure 4

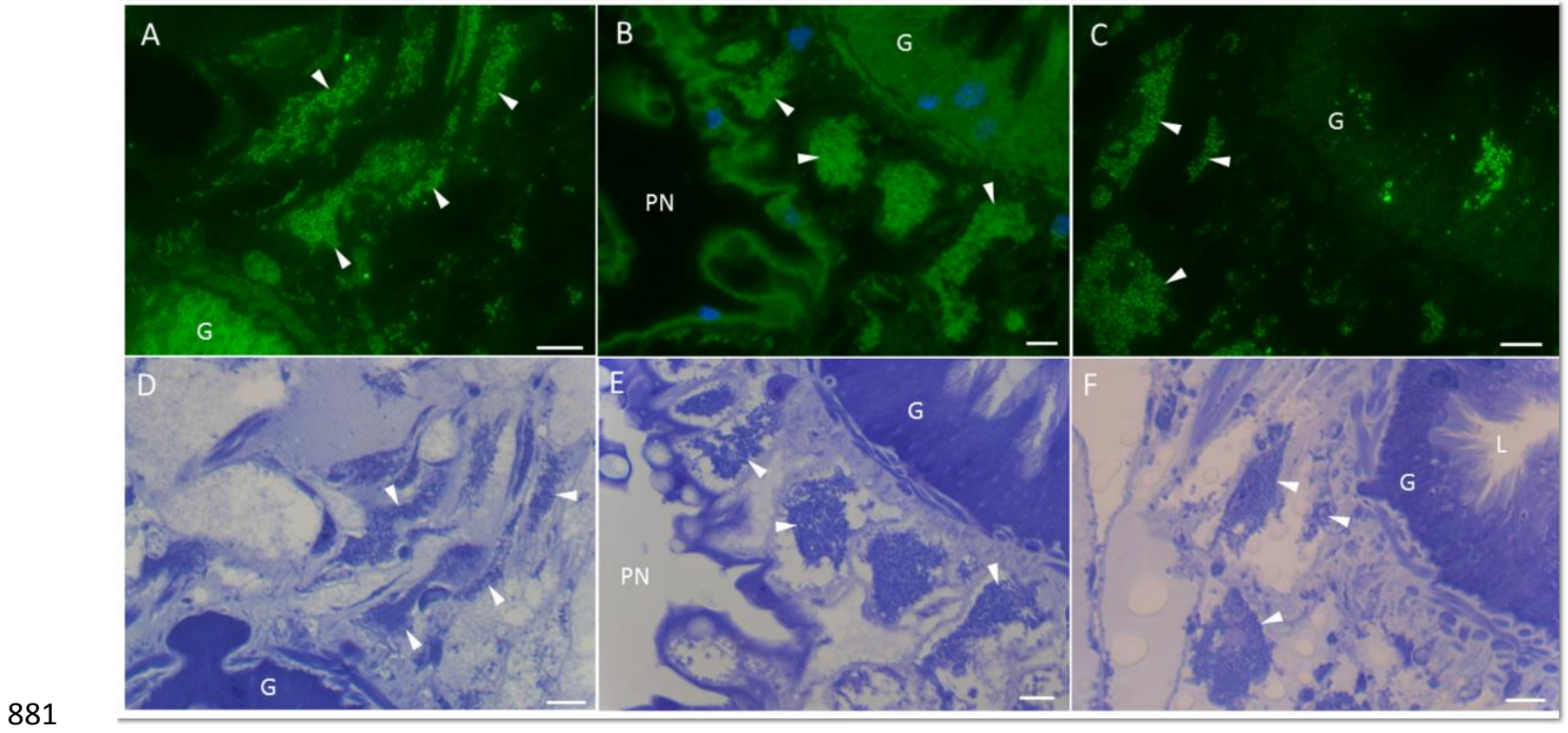

882

883 
$884 \quad$ Figure 5
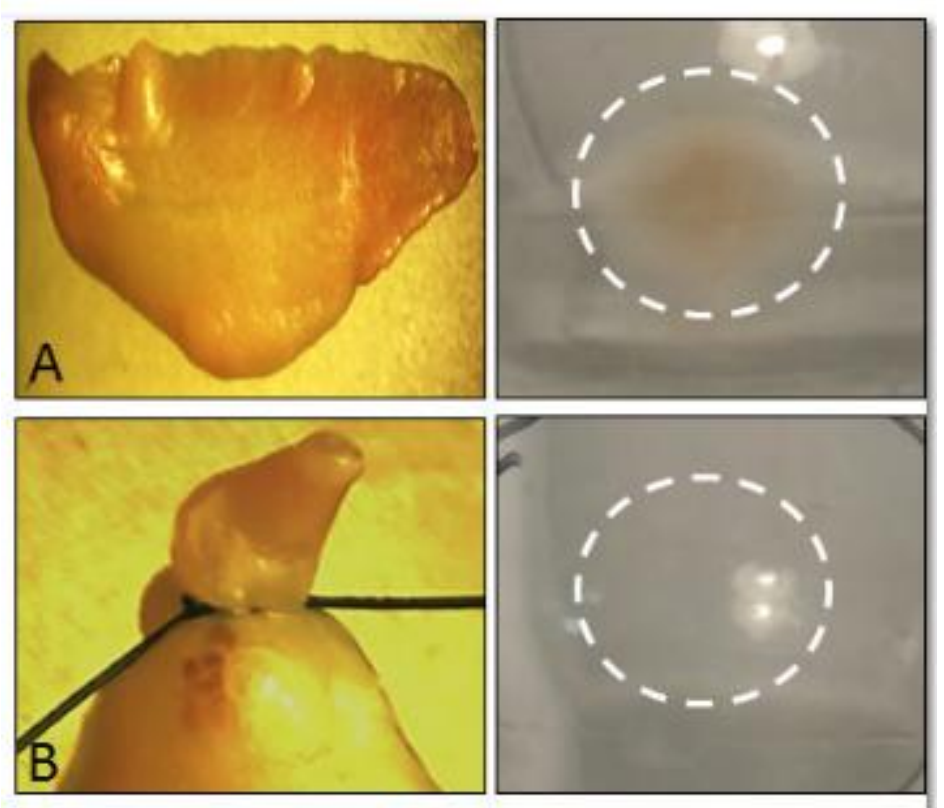

885
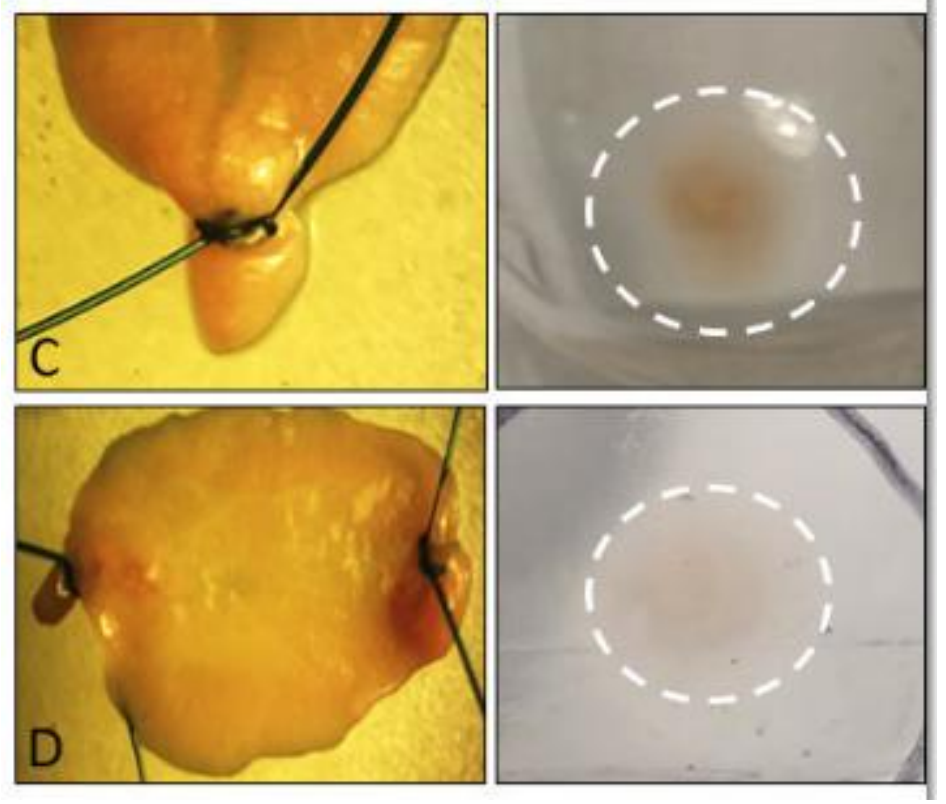

886

887 
Figure 6

$$
\text { AMPDL3 }{ }^{159} \text { Ctrl OSL }
$$

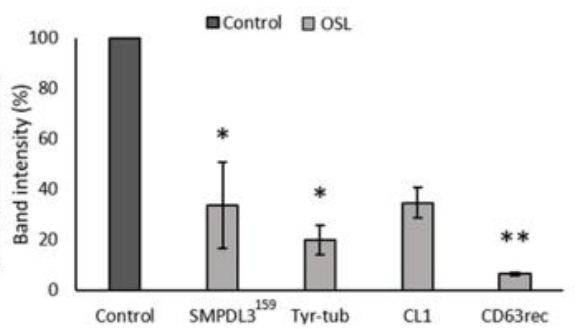

C

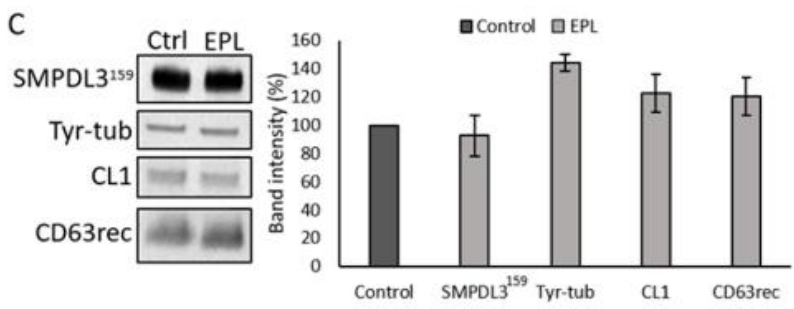

889 890 891 892
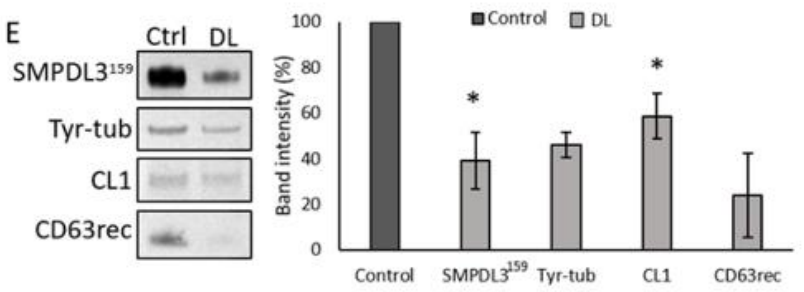

B

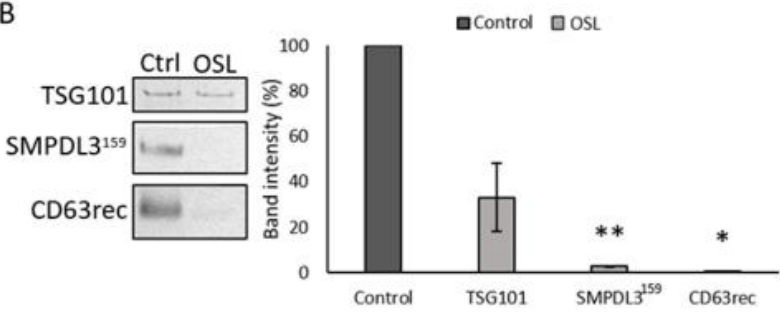

D
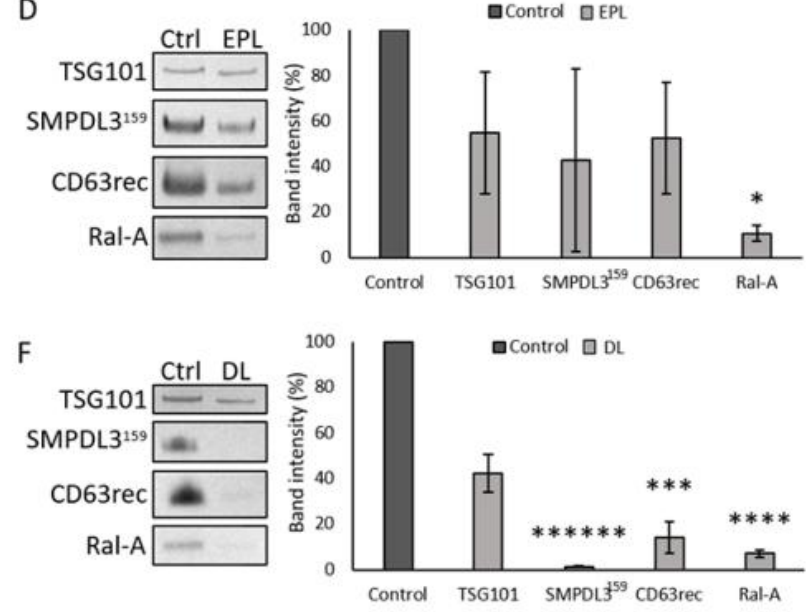
$893 \quad$ Figure 7

A

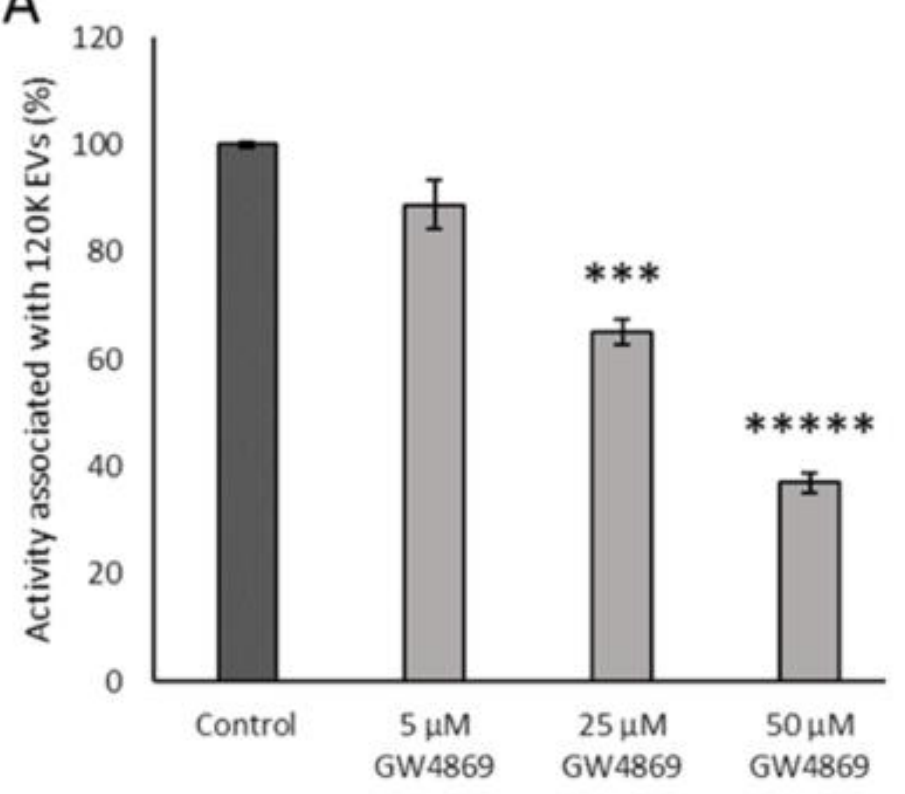

B

C
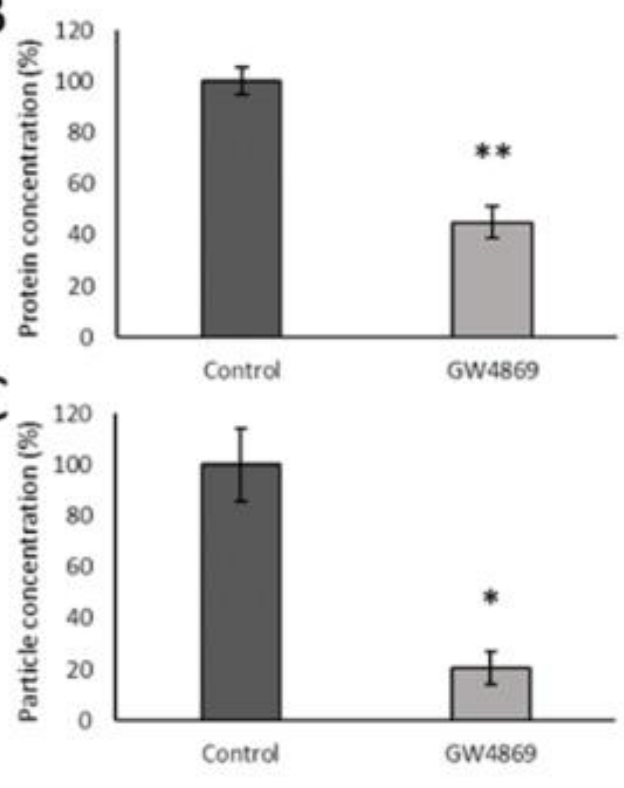

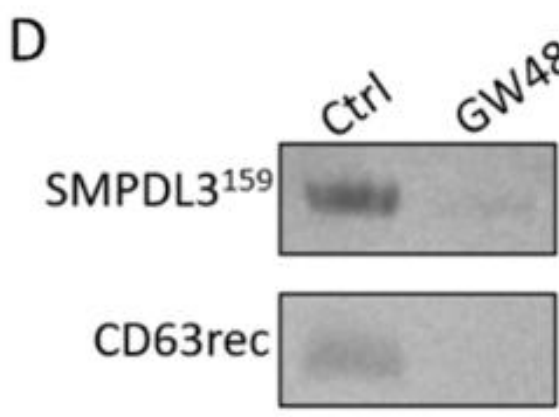

895

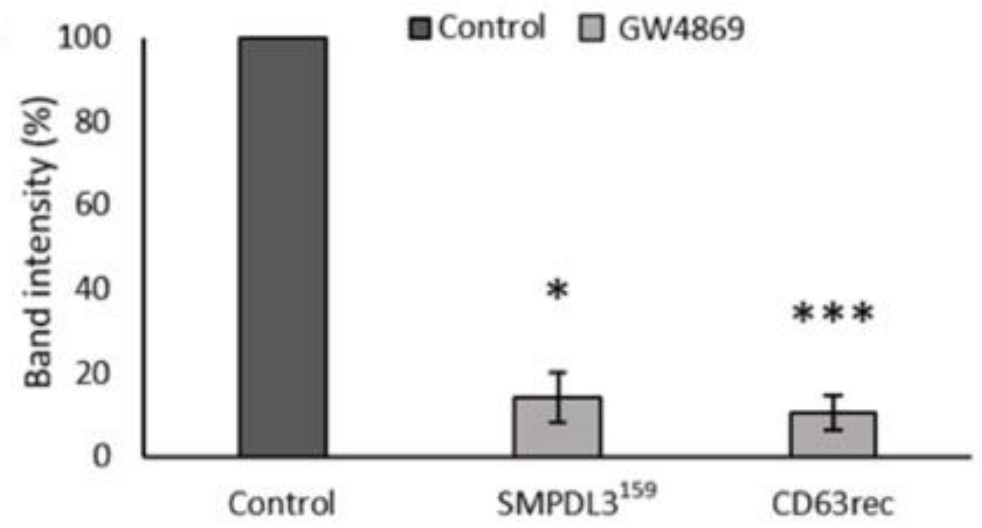

896 
$897 \quad$ Figure 8
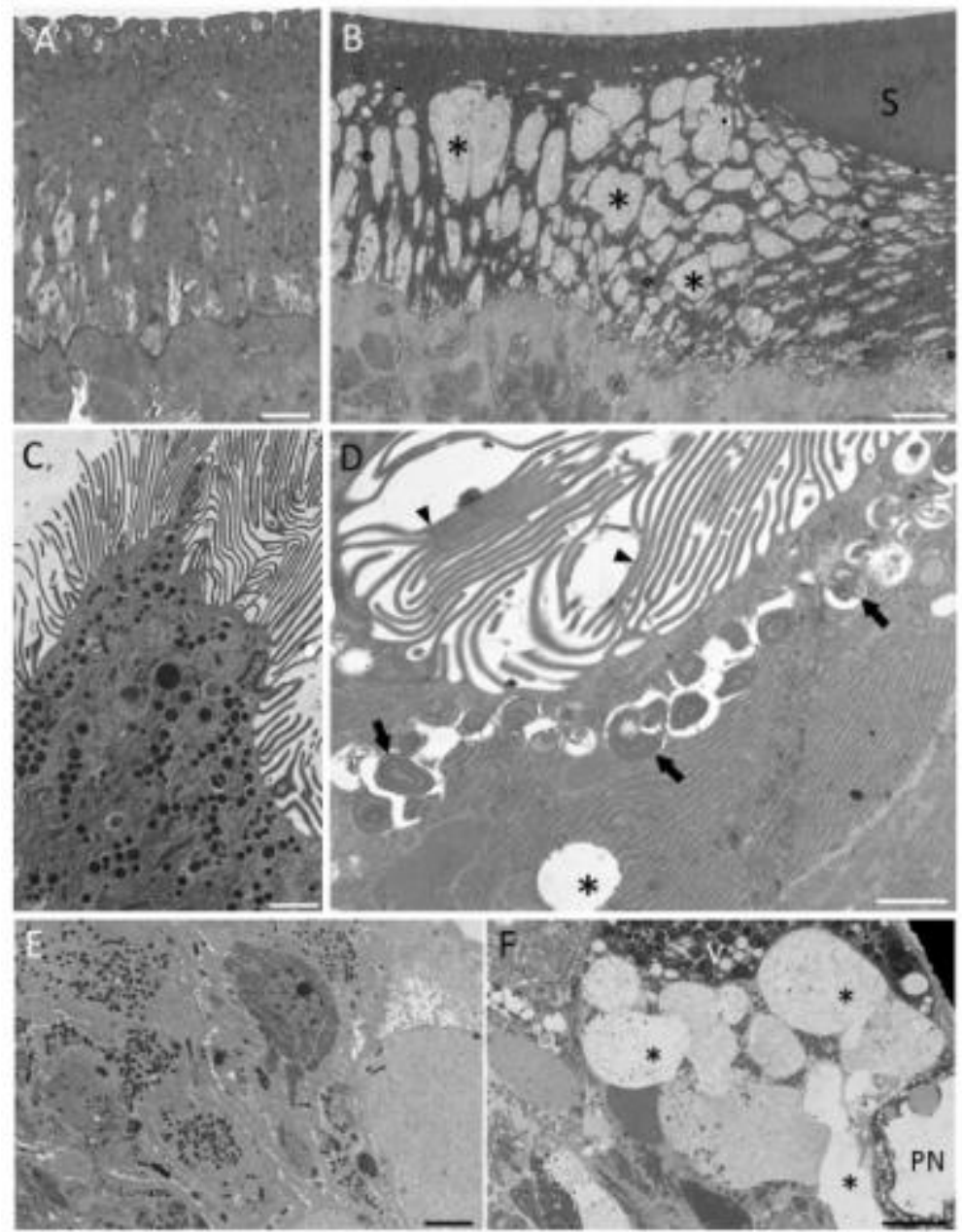

898

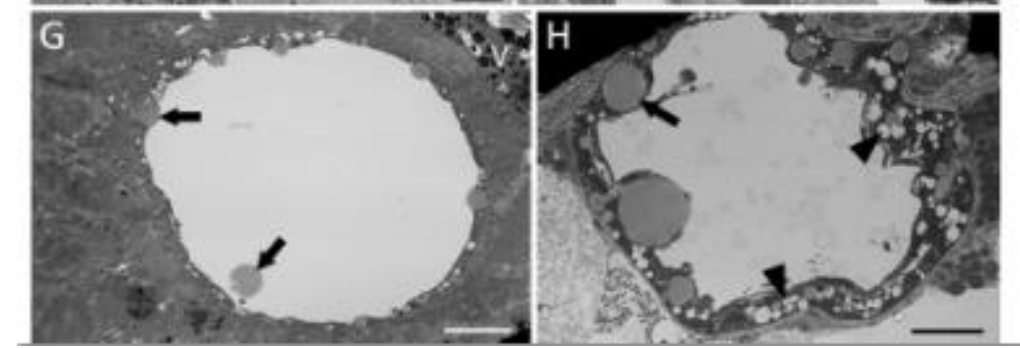

899 